\title{
Generalized Blob Algebras and Alcove Geometry
}

\author{
Paul P Martin 巴 and David Woodcock ${ }^{1}$
}

\section{Introduction}

Soergel has given a beautiful procedure 61, 60 for analysing tilting modules for quantum groups at roots of unity through parabolic Kazhdan-Lusztig polynomials. The procedure itself may be applied formally to an alcove geometry, without reference to representation theory. Hence it may be applied, in principle, in cases which are beyond the scope of Soergel's proof of representation theoretic interpretation. It is interesting then to try to find algebras for which the resultant combinatorial data has a representation theoretic interpretation, even though Soergel's proof is not applicable. The output of the usual procedure in type- $A$ may be mapped by Ringel duality 22 to the content of projective modules for certain quotients of ordinary Hecke algebras. (There it may be understood in terms of idempotent decompositions of 1 [53].) This leads to a determination of decomposition numbers for standard modules of the Hecke algebras themselves. Here we consider generalising the implementation of the procedure on this Ringel dual side. We do this by constructing generalized Hecke algebra quotients which (mildly) generalize the usual role of alcove geometry.

One example where the formal procedure gives the correct decomposition numbers is the blob algebra $b_{n}$ [52, 54] (a certain two parameter affine Hecke algebra quotient). We demonstrate the procedure for this example in $\$ 1.1$ below. There is a set of key properties of $b_{n}$ (see $\$ 4.2$ ), which it has in common with the ordinary Hecke algebra quotients (see $\$[.2$ ), which may serve to explain the phenomenon. In this paper we discuss generalisations of $b_{n}$ which also possess these properties.

To generalise $b_{n}$ suitably we first place it in the context of affine/cyclotomic Hecke and Ariki-Koike-Levy algebras [10, 2, 50] (although these are not themselves the generalisations we require). The study of these algebras is interesting both abstractly and also since they are useful in studying solutions to the reflection equation in integrable statistical mechanics (see [50] for references). This parallels the role of ordinary Hecke algebras in solving the Yang-Baxter equation. In both cases the 'physical' representation theory focuses attention on specific quotients, and implies that decomposition number data should be organized in a certain specific way. In the ordinary Hecke case this is the 'Soergel' rather than the 'LLT way' 42 (as complete data sets these are equivalent but computationally they are not 53]). Thus while the algebras we shall construct have representation theory which is accessible in principle by LLT methods [1], this does not remove the need for a generalized (dual) Soergel approach. In $\S 1.2$ we discuss quotients of affine Hecke algebras generalizing $b_{n}$ which, like $b_{n}$, realize certain key ingredients of this approach - in particular they possess a weight space. In $\$ 3$ we imbue this space with an alcove structure and

\footnotetext{
${ }^{1}$ Mathematics Department, City University, Northampton Square, London EC1V 0HB, UK.
} 
verify a linkage principle[35]. These generalized algebras are quotients of cyclotomic Hecke algebras by certain primitive and central idempotents. In $\S$ 国 we show how the simplest non-trivial such quotient may effectively be identified with $b_{n}$. The rest of the paper discusses outstanding technical issues in showing the validity of the generalized Soergel procedure for the generalized algebras (the primitive and central idempotents of the cyclotomic Hecke algebras are computed in \$5).

One property of the ordinary Hecke quotients not possessed by these generalisations is the defining representation on 'tensor space', realizing Ringel duality [21] with a quantum group quotient (cf. [19, 3, 59]). Such a faithful tensor space representation is not manifestly necessary for our purpose, but would be very useful. In $\$$ G we address this problem, culminating in the construction of some intriguing new concrete representations of $b_{n}$ which are candidates. (The serendipitous constructions of a number of other interesting new representations of $b_{n}$ and its generalisations are outlined in $\S \S 0$ 8.)

We will argue that the representation theory of these algebras, while containing that of ordinary Lie theoretic objects, is in a sense more simply described. Given that the representation theory of $b_{n}$ itself is known for $q$ root of unity in arbitrary charactersitic [15], the possibility that open questions in ordinary Lie representation theory may be accessible by this route makes these algebras particularly interesting objects for study.

\subsection{Alcove geometry and decomposition numbers for $b_{n}$}

We will recall the definition [54, §2] of the blob algebra $b_{n}=b_{n}(q, m)$ in $\S 4.2$. The decomposition numbers in the 'doubly critical' case ( $q$ a primitive $l^{\text {th }}$ root of $1 ; m$ an integer, $|m|<l$ - see $\S($ 何) in characteristic zero are determined in [54, §9] by algebraic methods. A formal application of Soergel's procedure to this case works as follows.

First recall, quite generally, that a Euclidean space with reflection hyperplanes removed has a set ( $\mathfrak{A}$, say) of connected components, called alcoves [35, Ch.6] [9]. For $s$ a reflection hyperplane and $B$ an alcove we denote by $B s$ the image of $B$ in $s$. Each nonempty intersection of the closure of an alcove with a hyperplane is called a wall of the alcove (here we will confuse each such wall with the hyperplane containing it). We make no assumption about the relation of hyperplanes to the origin 0 , except that the origin lies in the interior of an alcove, called the fundamental alcove and denoted $A^{0}$. Define $|B|$ as the number of hyperplanes between $B$ and 0 (so $\left|A^{0}\right|=0$ ).

In algebraic Lie theory one starts with a set of ordinary (non-affine) reflections generating the ordinary Weyl group. The $(q)$-group weight space is the underlying Euclidean space with its origin $\rho$-shifted [35]. In particular, even when an affine reflection is added the origin is at a fixed position at the base of the dominant region. In our case there is effectively no ordinary Weyl group and no dominant region, that is to say, the placement of all hyperplanes is controlled by parameters of the algebra. Thus the weight space for the blob algebra, just as for $s l_{2}$, is the Euclidean space associated to the $A_{1}$ Coxeter system, i.e. it is effectively $\mathbb{R}$ [54, $\S 6]$. However now, cf. $s l_{2}$, all integral weights are dominant, that is to say, simple modules may be indexed by $\mathbb{Z}$ (we shall explain this, in the context of our generalised construction, shortly). In the $b_{n}$ case, the reflection hyperplanes are just points, and those generating the affine Weyl group lie at $-m$ and $l-m$. (And an alcove $B \in \mathfrak{A}$ is a connected component of $\mathbb{R}$ with the reflection points removed.) A reflection is 
'upward' if $|B|<|B s|$ (cf. the usual $s l_{2}$ situation).

For each alcove $A$ one defines a map

$$
n_{A}: \mathfrak{A} \rightarrow \mathbb{Z}[v]
$$

where $v$ is a formal parameter, as follows. (For simplicity we ignore features of Soergel's procedure which do not arise in our case.) Firstly $n_{A}(A)=1$ and $n_{A}(B) \neq$ 0 implies $|B| \leq|A|$. Note that $n_{A^{0}}$ is determined immediately by this, and proceed inductively on $>$. For each alcove $A$ there will be a wall $s$ of $A$ such that $|A s|>|A|$. Each alcove $B$ has one wall which is in the affine Weyl orbit of $s$, and we will write $B . s$ for the image of $B$ in that wall (thus $A s=A . s$ ). Then, with $n_{A}$ known, set

$$
n_{A s}^{\prime}(B . s)= \begin{cases}n_{A}(B)+v^{-1} n_{A}(B . s) & |B . s|>|B| \\ v^{-1} n_{A}(B)+n_{A}(B . s) & |B . s|<|B|\end{cases}
$$

and define $n_{A s}$ by

$$
n_{A s}(C)=n_{A s}^{\prime}(C)-\left.\sum_{B:|B|<|A s|} n_{A s}^{\prime}(B)\right|_{v=0} n_{B}(C) .
$$

(That this procedure is well defined is not trivial [61].)

Evaluating $\left.n_{A}(B)\right|_{v=1}$, this construction is (formally) computing the standard module content of tilting modules in a Ringel dual algebra. (Any two weights which are in different affine Weyl orbits [35] are in different blocks [7, Ch.1]. Thus each block intersects each alcove in at most 1 weight and, fixing a block, it is the modules with these weights which $\left.n_{A}(B)\right|_{v=1}$ describes.) The corresponding data for $b_{n}$ is, in effect, the standard module content of projective modules. By reading by column instead of row, as it were, we convert this to the simple module content of standard modules [21] (truncation to a finite column interval, such as that pictured in the example which follows, corresponds to localisation to some finite $n$ - see ingredient I2 below). As noted, this construct is entirely formal, however in fact

Proposition. For $\lambda \in A$ and $\mu \in B$ the $b_{n}$ standard composition multiplicity

$$
[\Delta(\mu): L(\lambda)]=\left\{\begin{array}{ll}
\left.n_{A}(B)\right|_{v=1} & \mu \text { in the affine Weyl orbit of } \lambda ;|\lambda| \leq n \\
0 & \text { otherwise }
\end{array} .\right.
$$

Further, the power of the formal parameter $v$ determines the Loewy layer.

Proof: The composition multiplicity data is determined in [54, §9]. A table 
illustrating the computation of the polynomials $n_{A}(B)$ is as follows.

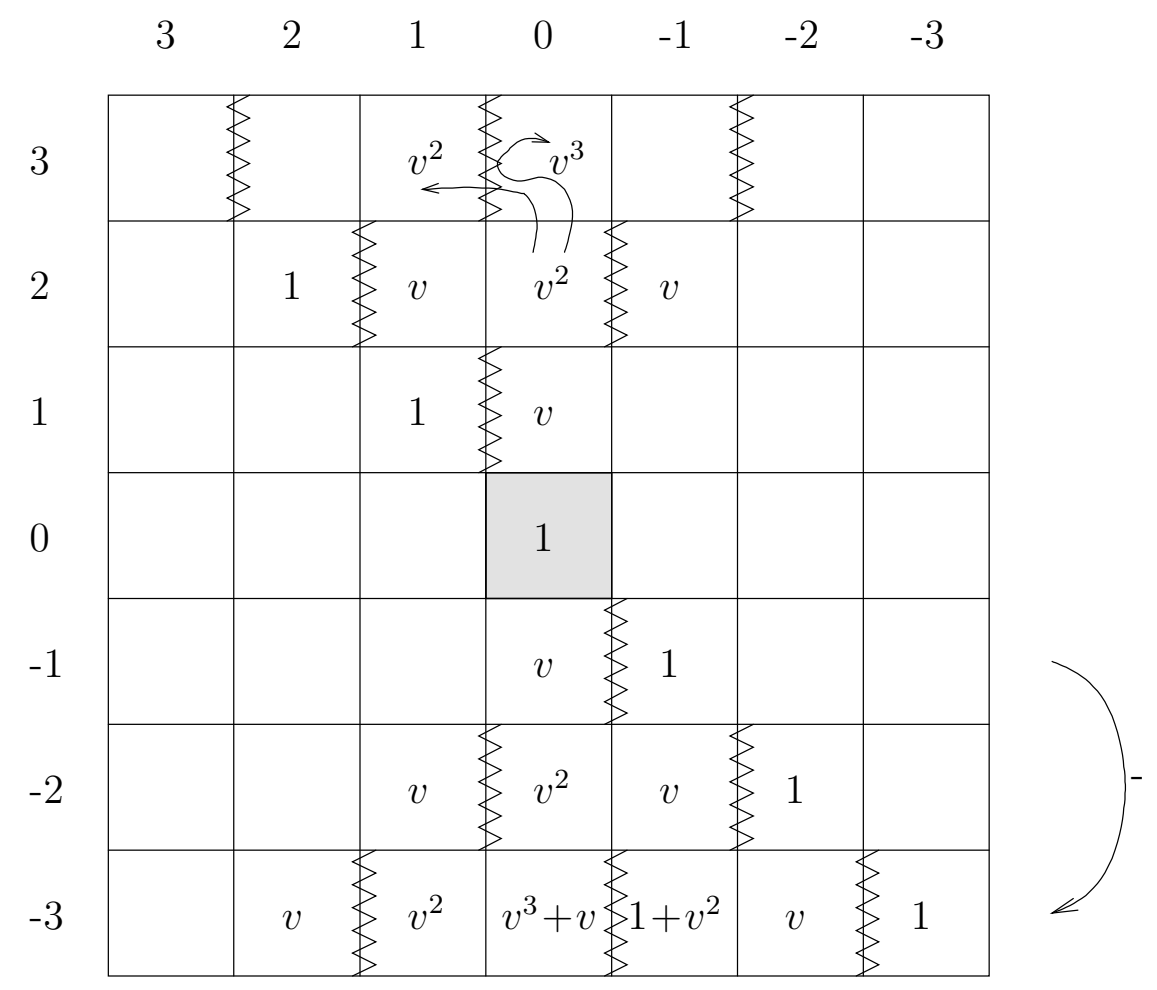

The row position in the table gives $A$ and the column position $B$; and $n_{A^{0}}\left(A^{0}\right)$ is shaded. If The table is complete for the rows shown, except the top and bottom rows. For the top row, the arrows within the table illustrate the contributions to the $n_{A s}^{\prime}$ from a particular $n_{A}(B)$ (the shaded lines are the relevant walls for reflection in each case). The arrow outside the table illustrates a required subtraction to obtain $n_{A}$ in the bottom row (which is complete except for this subtraction). The pattern is clear, and one sees immediately that the formal procedure reproduces the multiplicity and layer data.

The case $b_{n}(q, 1)$ contains the ordinary Ringel dual, $\operatorname{End}_{U_{q} s l_{2}}\left(V_{2}^{\otimes n}\right)$, as a quotient. Note that the ordinary Soergel procedure is embedded in this version (in the 'dominant' region of case $m=1$ ) accordingly.

The 'idempotent splitting' analysis described in [53] applies in principle in this situation, giving a heuristic explanation of why Soergel's procedure is relevant here. Following this paradigm there are a set of natural generalisations for which an analogous method should work.

The ingredients are (in precis, see also [53, 55]):

I1 A tower of unital algebras $A_{n} \subset A_{n+1}$ over a ring with indeterminates, and a multiplicity-free [66 semisimple specialisation [13] (split, and we will only consider characteristic 0 here).

For a tower as in $\mathrm{I1}$, let $\mathrm{B}_{\mathrm{A}_{-}}$denote the Bratteli diagram of the semisimple case. Regarded as a set, $\mathrm{B}_{\mathrm{A}_{-}}$will here mean the vertex set of this graph.

\footnotetext{
${ }^{2}$ The rows and columns, and hence the alcoves, are labelled using $\mathbb{Z}$. These labels should not be confused with points in the underlying space $\mathbb{Z}$ (each alcove contains $l-1$ such points) or with weights.
} 
I2 (i) A quasi-hereditary global limit via an idempotent $e \in \mathrm{A}_{m}$ (some $m$ ) and isomorphisms 28

$$
e \mathrm{~A}_{n+m} e \cong \mathrm{A}_{n}
$$

(and hence a tower of recollement [12]). (Exclude consideration of specialisations in which $e$ is not well defined.)

(ii) A map $\mathfrak{P}_{\mathrm{A}}$ from $\mathrm{B}_{\mathrm{A}_{-}}$to a global index set $\Lambda$, which localises at each $n$ to an index set $\Lambda(n)$ for standard modules $\Delta(\mu)$ p of $\mathrm{A}_{n}$ (i.e., such that $\Lambda(n) \hookrightarrow \Lambda(n+m)$ via the full embedding of $\mathrm{A}_{n}-\bmod$ in $\mathrm{A}_{n+m}-\bmod$ consequent on eqn.(1), while $\left.\dot{\cup}_{n} \Lambda(n) \cong \mathrm{B}_{\mathrm{A}_{-}}\right)$.

Let $\operatorname{Res}(\mu) \subset \Lambda$ denote the set of weights of standard factors of the restriction $\operatorname{Res}_{\mathrm{A}_{n}} \mathrm{~A}_{n+1} \Delta(\mu)$, and $\operatorname{Ind}(\mu)$ of the corresponding induction.

I3 A space $V$ (for definiteness we will assume this is a real Euclidean space) and map $\Lambda \hookrightarrow V$ with the following properties. The convex hull of $\operatorname{Res}(\mu)$ intersects $\Lambda$ in a subset of $\{\mu\} \cup \operatorname{Ind}(\mu) \cup \operatorname{Res}(\mu)$ (locality of induction and restriction); the set $\rho_{\mu}$ of reflections in $V$ which fix $\{\mu\}$ and $\operatorname{Res}(\mu)$ fixes $\Lambda$; the group $W$ generated by $\cup_{\mu} \rho_{\mu}$ is an affine Weyl group [33, §4.2]; and $\Lambda$ is a subset of the set of point facets in the alcove geometry induced by $W$ on $V$.

I4 Control of bases for the algebras and standard modules - including the means in principle to compute Gram matrices in the case of indeterminate parameters.

I5 Explicit forms for the simplest primitive idempotents (in particular any primitive and central idempotents).

I6 A linkage principle [35] $-\Delta(\mu), \Delta(\nu)$ are in different blocks if there does not exist any $w \in W_{l}$ ( $W_{l}$ a suitably rescaled version of $W$, depending on the specialisation) such that $w \mu=\nu$.

\subsection{Role and realization of ingredients 1, 2(ii) and 3}

Recall [43] (and cf. [41]) the affine Hecke algebra $H(n)$ defined by generators $\left\{1, X, g_{1}, \cdots, g_{n-1}\right\}$ and relations

$$
\begin{array}{ccc}
g_{i} g_{i \pm 1} g_{i}=g_{i \pm 1} g_{i} g_{i \pm 1} & g_{i} g_{j}=g_{j} g_{i} & i \neq j \pm 1 \\
g_{1} X g_{1} X=X g_{1} X g_{1} & g_{j} X=X g_{j} & j>1 . \\
\left(g_{i}-q\right)\left(g_{i}+q^{-1}\right)=0 &
\end{array}
$$

The cyclotomic Hecke algebra [10] $\mathrm{H}=\mathrm{H}(n, d)$ is the quotient $\Psi_{d}$ of $H(n)$ by

$$
\prod_{i=1}^{d}\left(X-\lambda_{i}\right)=0 .
$$

Here $q, \lambda_{1}, \cdots, \lambda_{d}, \cdots$ are parameters, which we may begin by regarding as indeterminates. Write $\mathcal{A}$ for $\mathbb{Z}\left[q, q^{-1}, \lambda_{1}, \ldots, \lambda_{d}\right]$ and $\mathcal{K}$ for the quotient field. Write $\mathrm{H}^{\mathcal{A}}(n, d)$ for $\mathrm{H}(n, d)$ over $\mathcal{A}$. This is a free module over $\mathcal{A}$ [2] (see $\oint_{2}$ ). The case

\footnotetext{
${ }^{3}$ Standard in the quasi-hereditary sense, but we might also hope these modules are 'nice' in some Kazhdan-Lusztig sense 40, 24.
} 
$d=2, \lambda_{1}=-\lambda_{2}^{-1}$ is essentially the $B$-type Hecke algebra (cf. [32, 48]). For any $d^{\prime}>d$ let $\Psi_{d}: \mathrm{H}\left(n, d^{\prime}\right) \rightarrow \mathrm{H}(n, d)$ also denote the quotient by equation(5). Denote by $\mathrm{H}(-, d)$ the sequence of inclusions

$$
\mathrm{H}(n, d) \subset \mathrm{H}(n+1, d)
$$

Usually we will fix an $\mathcal{A}$-algebra $k$ which is a field, and which as a field is $\mathbb{C}$, and consider $\mathrm{H}(n, d)=\mathrm{H}^{\mathcal{A}}(n, d) \otimes_{\mathcal{A}} k$. The semi-simple generic structure of $\mathrm{H}(n, d)$ over $\mathbb{C}$ is well known, through that of the specialisation to the group algebra of the group $Z_{d} 2 S_{n}$ (confer [34, 32, 13] as in [2]). We recall it briefly. An integer partition $\mu$ of degree $n$ is a list $\left(\mu_{1}, \mu_{2}, \cdots\right)$ of non-negative integers such that $\mu_{i} \geq \mu_{i+1}$ and $\sum_{i} \mu_{i}=n$. There is a natural correspondence with Young diagrams of degree $n$. Denote by $\Lambda_{n}=\Lambda_{n}^{d}$ the set of ordered lists of $d$ integer partitions, of summed degree $n$ (called $d$-partitions of degree $n$ ). For example

$$
\Lambda_{2}^{2}=\left\{((2), 0),\left(\left(1^{2}\right), 0\right),((1),(1)),(0,(2)),\left(0,\left(1^{2}\right)\right)\right\} .
$$

The conjugacy classes of $Z_{d} 2 S_{n}$ are readily seen to be indexed by $\Lambda_{n}^{d}$ [44, 55], thus $\mathrm{H}(n, d)$ has simple modules $\Delta_{\mu}$ indexed by $\mu \in \Lambda_{n}^{d}$. Similarly the Bratteli diagram $\mathrm{B}=\mathrm{B}_{\mathrm{H}_{(-, d)}}$ of the natural tower of semi-simple algebras $\mathrm{H}(-, d)$ is determined by

$$
\operatorname{Res}_{\mathrm{H}(n, d)}^{\mathrm{H}(n+1, d)} \Delta_{\mu}=\bigoplus_{i=1}^{d} \bigoplus_{j} \Delta_{\mu-e_{j}^{i}}
$$

where the sum over $j$ is over possible subtractions of one box from the $i^{\text {th }}$ Young diagram of $\mu$.

For each $n>1$, there are $2 d$ one dimensional irreducible representations $R_{ \pm l}$ $(l=1,2, \cdots, d)$ of $\mathrm{H}(n, d)$, given by $R_{ \pm l}(X)=\lambda_{l}$ and $R_{ \pm l}\left(g_{i}\right)= \pm q^{ \pm 1}$. The representation $R_{l}$ corresponds to the module $\Delta_{\mu}$ with multipartition $\mu=\left(,, \mu_{l},,\right)$ in which all component integer partitions are empty except the $l^{\text {th }}$ partition, which is either $(n)$ (case $l>0$ ) or $\left(1^{n}\right)$ (case $\left.l<0\right)$. For each $n$ we may associate an unique primitive (and central) idempotent to each of these representations, in the algebra over generic $k$ 31]. We write these idempotents $e_{n}^{ \pm l}$. For $l \in\{1,2, \ldots, d\}$ the element $e_{n}^{ \pm l}$ of $\mathrm{H}(n, d)$ uniquely obeys

$$
\left(g_{i} \mp q^{ \pm 1}\right) e_{n}^{ \pm l}=0 \quad(i=1,2, \cdots, n-1), \quad\left(X-\lambda_{l}\right) e_{n}^{ \pm l}=0, \quad\left(e_{n}^{ \pm l}\right)^{2}=e_{n}^{ \pm l} .
$$

The inclusion (6) allows us to regard $e_{n}^{ \pm l}$ as an idempotent in $\mathrm{H}(n+1, d)$, albeit neither primitive nor central in general. Indeed the idempotent will be expressible as a sum of primitive idempotents in reciprocity with the rule (77).

In $\oint$ of this paper we give explicit formulae for all $e_{n}^{ \pm l}$ for all $d$. For now we will be concerned particularly with $e^{ \pm l}:=e_{2}^{ \pm l}$. The reason for this is the desire for a small but significant generalisation of the set of dominant weights and the weight spaces underlying Soergel's procedure for case $A_{m-1}$ (i.e. $U_{q} s l_{m}$ ). Although the induction and restriction rules are straightforward, and satisfy ingredient 1 , the 'weight space' of $\mathrm{H}(-, d)$ (in which distance $d(\mu, \lambda)$ is the minimum number of steps on B from $\mu$

${ }^{4}$ Let $1=\sum_{\mu} e^{\mu}$ be the unique decomposition of 1 into primitive central idempotents of $\mathrm{H}(n+1, d)$. Then $e_{n}^{ \pm l}=\sum_{\mu} e_{n}^{ \pm l} e^{\mu}$ is this decomposition; i.e. it is also unique, even though the decomposition of 1 into primitive idempotents is not. 
to $\lambda$ ) is somewhat unmanageable geometrically, cf. our desired ingredients 2 and 3 . What is wanted is something like an analogue for $\mathrm{H}(-, d)$ of the quotients

$$
H_{n}^{m} \cong \operatorname{End}_{U_{q} s l_{m}}\left(V_{m}^{\otimes n}\right)
$$

of the ordinary Hecke algebra $H_{n}$. The Bratteli diagram $\mathrm{B}_{H_{-}}$of the ordinary Hecke algebra is the Young tree, but via (9) the quotients $H_{n}^{m}$ are the natural incarnations of the Ringel duals of $A_{m-1}$ quantum groups, and hence may be associated to the same weight spaces, and satisfy ingredients $1-6$. Let us briefly review this. Let $e_{m+1}^{ \pm}$ denote the two primitive and central idempotents of $H_{m+1}$ (for simplicity assume $[m+1] ! \neq 0$ for now $)$. The $H_{n}^{m}$ are such that

$$
0 \longrightarrow H_{n} e_{m+1}^{-} H_{n} \longrightarrow H_{n} \longrightarrow H_{n}^{m} \longrightarrow 0
$$

is exact. For $[m] ! \neq 0$ they are quasi-hereditary and satisfy ingredient 2 , for example, through

$$
e_{m}^{-} H_{n+m}^{m} e_{m}^{-} \cong H_{n}^{m}
$$

Let $\Lambda^{1, m}$ denote the set of Young diagrams of $<m$ rows, regarded as a subset of $\mathbb{Z}^{m-1}$. Let $v=(1,1, \ldots, 1) \in \mathbb{Z}^{m-1}$ and let $\mathbb{Z}^{m-1} / v$ denote the corresponding quotient set. Note that the injective map from $\mathbb{Z}^{m-2}$ into $\mathbb{Z}^{m-1}$ given by $\left(\mu_{1}, \mu_{2}, \ldots, \mu_{m-2}\right) \mapsto$ $\left(\mu_{1}, \mu_{2}, \ldots, \mu_{m-2}, 0\right)$ has image a set of representative elements of $\mathbb{Z}^{m-1}$ in $\mathbb{Z}^{m-1} / v$. Denote by $\mathfrak{P}^{m-1}$ the corresponding surjective map from $\mathbb{Z}^{m-1}$ to $\mathbb{Z}^{m-2}$ (and also its restriction to $\Lambda^{1, m}$, whose image is $\Lambda^{1, m-1}$ ). Note that $\mathrm{B}_{H_{-}^{m}}$, the set of weights for $H_{n}^{m}$ for all $n$, is $\Lambda^{1, m+1}$. In the sense of ingredient 2(ii) the set of isomorphisms (11) collapses $\mathrm{B}_{H_{-}^{m}}$ into $\Lambda^{1, m}$, which is the set of dominant weights of $s l_{m}$, via $\mathfrak{P}^{m+1}$.53]. That is $\mathfrak{P}_{H^{m}}=\mathfrak{P}^{m}$; and $\Lambda^{1, m}(n)$, the index set for $H_{n}^{m}$, is the subset of $\Lambda^{1, m}$ of diagrams of degree $\leq n$ and congruent to $n$ modulo $m$.

Delightfully, we find that only the $n=2$ idempotents are needed for an analogue of equation(10) for $\mathrm{H}(n, d)$ (see also [55]). Denote the sum of the ideals generated by $\left\{e_{2}^{-l} \mid\right.$ all $\left.l\right\}$ by

$$
\mathcal{D}_{d}=\mathcal{D}_{d}(n):=+_{l=1}^{d} \mathrm{He} e_{2}^{-l} \mathrm{H}
$$

(we will modify this definition very slightly later). Define algebra $H^{\mathcal{D}}=H^{\mathcal{D}}(n, d)$ by $H^{\mathcal{D}}(1, d)=\mathrm{H}(1, d)$ and for $n>1$ by exactness of the sequence

$$
0 \longrightarrow \mathcal{D}_{d} \longrightarrow \mathrm{H} \longrightarrow H^{\mathcal{D}} \longrightarrow 0 \text {. }
$$

The idea is to restrict consideration to the subset $\Lambda^{\mathcal{D}}$ of $\mathrm{H}(-, d)$-weights in which each integer partition is the trivial partition of that degree. Such an $\mathrm{H}(-, d)$-weight is characterised by a sequence of $d$ non-negative integers, i.e. the degrees of the component integer partitions (in this way we have an action of $\mathfrak{P}^{d}$ on $\Lambda^{\mathcal{D}}$ ). This sequence need not be ordered as an integer partition, and hence the set of such weights maps onto the set of all weights of $A_{d-1}$ (i.e. not just the usual dominant weights). For example with $d=3$, the weight $((2),(4),(3))$ becomes $(2,4,3)$, and 
$\mathfrak{P}^{3}((2,4,3))=(-1,1):$

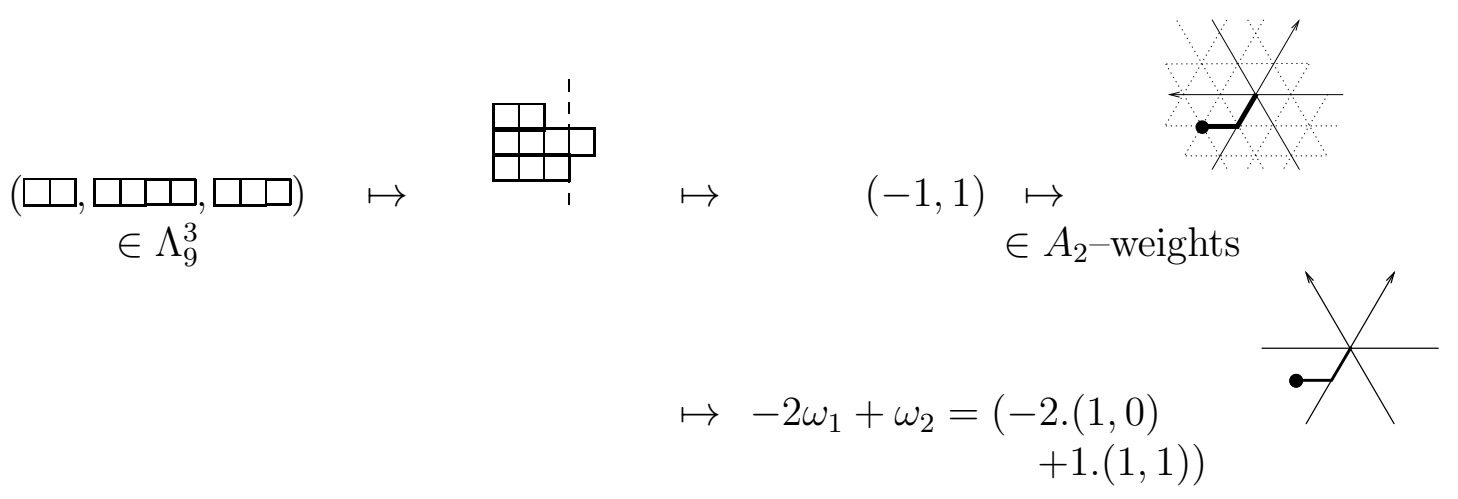

(the second row just shows the same weight in terms of fundamental weights); while with $d=2$

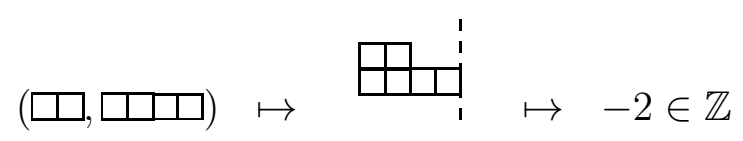

this last, note, being the weight set used in $\S 1.1$. Note that $\Delta_{\mu} \in \mathrm{H}-\bmod$ is also in $H^{\mathcal{D}}$-mod if and only if $\mu \in \Lambda^{\mathcal{D}}$ (in which case, as an $H^{\mathcal{D}}$-module we write it $\left.\Delta\left(\mathfrak{P}^{d}(\mu)\right)\right)$. To see this note, from (7), that the restriction of $\Delta_{\mu}$ to $n=2$ contains a copy of one of the excluded one dimensional modules (not necessarily a direct summand in general) if and only if $\mu$ contains at least one integer partition with a second part (i.e. a Young diagram with a second row).

By construction then, the tower $H^{\mathcal{D}}(-, d)$ has ingredients 1,3 (restriction is local via (7) and induction via Frobenius reciprocity) and, at least formally, the final part of 2. Of course the construction is most interesting if it can be made to include non-semisimple specialisations (else the fundamental alcove of $\S 1.1$ is the whole weight space and the Soergel procedure is trivial). In the remainder of this paper we address ingredients $2,4,5$ and 6 from this point of view. In particular we identify the $d=2$ case with the blob algebra. This has useful implications for all $d>2$. Note that, fixing $k$, there are a number of distinct ways to impose a quotient relation of the form of $\Psi_{d}$ on $\mathrm{H}\left(n, d^{\prime}\right)$, corresponding to the choice of factors to remove in the strengthening of the relation (5). For $\lambda^{\prime}=\left(\lambda_{1}, \lambda_{2}, \ldots, \lambda_{d^{\prime}}\right)$ and $\lambda$ any subsequence of this of length $d^{\prime}-d$ let $\Psi_{d}^{\lambda}$ denote the strengthening by omission of the factors $\left(X-\lambda_{i}\right)$ with $\lambda_{i}$ in $\lambda$. The quotient $\Psi_{d}^{\left(\lambda_{i}\right)}$ commutes with the quotient to $H^{\mathcal{D}}$ so

Proposition. Fixing $k$, there are $d+1$ ways $\left(\Psi_{d}^{\left(\lambda_{i}\right)}, i=1,2, . ., d+1\right)$ to quotient to pass from $H^{\mathcal{D}}(n, d+1)$ to $H^{\mathcal{D}}(n, d)(n>0)$.

Returning to the example of $d=3$, we see that as $n$ varies the image of $\Psi_{2}^{\left(\lambda_{i}\right)}$ sweeps out a $2 \pi / 3$ radian arc of weight space (there will be a picture illustrating this in section 5.2), with the union of these arcs over $i=1,2,3$ giving the complete space. If $k$ gives a non-semisimple specialisation then, as we will see, there is at least one $i$ such that the corresponding tower of blob (i.e. $d=2$ ) algebras is critical (in the sense of [52, 54], or $\delta[1.1$, i.e. it has one or more reflection points. As $n$ varies a given $d=2$ reflection point sweeps out a straight line in this arc in $d=3$ weight space (see $\$ 5.2$ for details). This is then a reflection line of the $d=3$ alcove geometry, to which the Soergel procedure may be applied.

Our approach to ingredient 2 is through representation theory, and the last part of the paper addresses this. It includes a 'walk though' review of some earlier work 
in statistical mechanics which explains our approach to this problem, and concludes by defining certain representations of $b_{n}$ (and $H^{\mathcal{D}}(n, d)$ ) which are candidates for 'tensor space' representations (i.e. they would establish ingredient 2 if faithful, see $\$ 6$ - the question of faithfulness is not resolved here).

\section{Preliminaries}

Let involution $t: \mathbb{Z}\left[q, q^{-1}\right] \rightarrow \mathbb{Z}\left[q, q^{-1}\right]$ be given by $t(q)=-q^{-1}$; and let $s$ act on $\mathbb{Z}\left[\lambda_{1}, \cdots, \lambda_{d}\right]$ by permuting the indices on the $\left\{\lambda_{i}\right\}$ cyclically. Let $[n]=q^{n-1}+q^{n-3}+$ $\cdots+q^{1-n}$.

For $A$ an algebra, let $Z(A)$ denote the centre of $A$; and for $B \subseteq A$ let $Z_{B}(A)$ denote the centralizer of $B$ in $A$ (so $Z_{B}(A) \supseteq Z(A)$ and $Z_{A}(A)=Z(A)$ ).

(2.1) For $a=\left(a_{1}, a_{2}, \cdots, a_{n}\right)$ an $n$-tuple of natural numbers, the Weyl orbit of $a$ in $\mathbb{N}^{n}$ is the orbit of the $S_{n}$ action permuting indices. Let $Y=\left(Y_{1}, \ldots, Y_{n}\right)$ be an $n$-tuple of variables in some commutative ring $R$. Define

$$
Y^{a}=\prod_{i=1}^{n} Y_{i}^{a_{i}}
$$

and the monomial symmetric polynomial (see e.g. [23])

$$
\left(Y^{a}\right)^{\Sigma}=\sum_{a^{\prime}} Y^{a^{\prime}}
$$

where the sum is over all elements in the Weyl orbit of $a$.

(2.2) Let $R$ be a unique factorisation domain, $A$ a free $R$-module with basis $\mathbf{A}$, and $K$ the field of fractions of $R$. Consider any $e \in A \otimes_{R} K$ and let $a_{e} \in A$ be such that $\mathbf{e}=a_{e} e \in A \subset A \otimes_{R} K$. If there exists an $a_{e}$ such that the coefficient of some $\mathbf{a} \in \mathbf{A}$ in $\mathbf{e}$ is 1 , then $a_{e}$ and $\mathbf{e}$ are unique with this property up to a unit. If $A$ is an $R$-algebra and $e$ and idempotent, call such an e a preidempotent, and $a_{e}$ the corresponding normalisation of $e$.

Now let $R$ above be $\mathcal{A}$, and consider $A$ as a collection of $\mathbb{C}$-algebras by specialisation. We say that a property holds generically if it holds on an (Zariski) open subset of parameter space, and that it holds usually if the condition for failure to hold may be expressed as a single finite polynomial in a single variable (e.g. [2] is generically and usually invertible, $\left(q^{2} \lambda_{1}-\lambda_{2}\right)$ is generically but not usually invertible).

Let $e=\frac{\mathbf{e}}{a_{e}^{\prime}}$ be any explicit expression for an element in $A$ over $\mathcal{K}$ as above. For any point $x$ in parameter space (i.e. any $k$ ) there is an open region with $x$ in its closure in which the polynomial $a_{e}^{\prime}$ has no root, so $e$ may be evaluated as a limit at $k$. This process does not guarantee a unique finite limit. However, if $e$ is a primitive and central idempotent then any two finite limits must be the same, since they will have the properties of a unique primitive and central idempotent in $A$ over $k$. (I.e., they will induce the same simple projective module, $A \mathbf{e} \otimes k$.)

(2.3) Let $\sigma_{i}$ denote the elementary transposition $\sigma_{i}=(i i+1) \in S_{n}$, so $\sigma_{i}(i)=i+1$ and so on [31]. Let $\mathfrak{B}_{n}$ be a maximal set of inequivalent reduced words in the generators $\left\{g_{1}, \ldots, g_{n-1}\right\}$ of $H_{n}$. For each $w \in \mathfrak{B}_{n}$ note that there is a natural (reduced expression for an) element of $S_{n}$ associated to it by substituting $g_{i} \leadsto \sigma_{i}$. 
(2.4) Let

$$
X_{1}:=X \quad X_{j}:=g_{j-1} X_{j-1} g_{j-1}
$$

so in $H(n)$

$$
\begin{gathered}
{\left[X_{j}, X_{k}\right]=0} \\
{\left[X_{j}, g_{k}\right]=0 \quad j \neq k, k+1} \\
g_{k} X_{k+1}=X_{k} g_{k}+\left(q-q^{-1}\right) X_{k+1} \\
g_{k} X_{k}=X_{k+1} g_{k}-\left(q-q^{-1}\right) X_{k+1}
\end{gathered}
$$

and so

$$
\left[X_{k}+X_{k+1}, g_{k}\right]=\left[X_{k} X_{k+1}, g_{k}\right]=0
$$

Now

$$
X_{k}^{j}+X_{k+1}^{j}=\left(X_{k}^{j-1}+X_{k+1}^{j-1}\right)\left(X_{k}+X_{k+1}\right)-\left(X_{k}^{j-2}+X_{k+1}^{j-2}\right) X_{k} X_{k+1}
$$

so

$$
\left[g_{i},\left(X^{a}\right)^{\Sigma}\right]=0
$$

for all $i$ and any $a$.

(2.5) Let $X^{\Sigma}$ denote the algebra of symmetric polynomials in the $X_{i}$ s. Evidently $X^{\Sigma} \subseteq Z(H(n))$, and in fact Bernstein has noted that this is an equality (see appendix).

(2.6) It follows from equations (14 - 16) that any product of generators of $\mathrm{H}(n, d)$ can be expressed as a $\mathbb{Z}\left[q, q^{-1}, \lambda_{1}, \ldots, \lambda_{d}\right]$-linear combination of words from the set

$$
\mathfrak{C}_{n}^{d}=\left\{X^{a} w \mid a \in\{0,1, \cdots, d-1\}^{n}, w \in \mathfrak{B}_{n}\right\}
$$

The dimension of this spanning set is clearly $d^{n} n$ !, which is also the dimension of $\mathrm{H}(n, d)$, thus

Proposition. [2] The set $\mathfrak{C}_{n}^{d}$ is a basis for $\mathrm{H}(n, d)$.

Linearly extend $s$ and $t$ to act on $\mathrm{H}(n, d)$, fixing $\mathfrak{C}_{n}^{d}$ pointwise.

(2.7) Proposition. [55] Let $B$ be a basis for $H(n-1, d), D$ a basis for $\langle X\rangle$ and $G=g_{1} g_{2} \ldots g_{n-1}$. Then

$$
\left\{a G b, g_{1} a G b, g_{2} g_{1} a G b, \cdots, g_{n-1} \cdots g_{2} g_{1} a G b \mid a \in D, b \in B\right\}
$$

is a basis for $\mathrm{H}(n, d)$.

(2.8) Fix $d$ and set $p_{n}^{l}=\prod_{i \neq 1}\left(q^{2 n-2} \lambda_{l}-\lambda_{i}\right)$. Note that

$$
z_{n}^{l}=\prod_{k=1}^{n}\left(\prod_{i \neq l}\left(X_{k}-\lambda_{i}\right)\right)
$$

lies in $Z(\mathrm{H}(n))$ and obeys $\Psi_{d}\left(\left(X-\lambda_{l}\right) z_{n}^{1}\right)=0$. Comparing with (\&) we thus have

$$
e_{n}^{ \pm l}=\frac{z_{n}^{l}}{\prod_{k=1}^{n} p_{k}^{l}} e_{n}^{ \pm} .
$$

It follows that $\mathbf{e}_{n}^{ \pm l}=\left(\prod_{k=1}^{n}\left([k] p_{k}^{l}\right)\right) e_{n}^{ \pm l}$ is a preidempotent for $e_{n}^{ \pm l}$ (compare with the basis $\left.\mathfrak{C}_{n}^{d}\right)$. Now define

$$
\mathcal{D}_{d}=\mathcal{D}_{d}(n):=+_{l=1}^{d} \mathrm{He}_{2}^{-l} \mathrm{H}
$$

(a modification of the definition in section 1.2) and $H^{\mathcal{D}}$ accordingly. We will give another expression for $e_{n}^{ \pm l}$ shortly. 


\section{$3 \quad$ Standard modules and linkage}

The generators and relations in equations(2) (and inverses) define the ordinary braid group $A_{n}$-braid. Denote by $B_{n}$-braid the extension by $g_{0}=X$ (and inverse) obeying equations(3) (cf. [32, 49] and references therein). Thus $H(n)$ is a quotient of $\mathbb{C} B_{n}$-braid by the quadratic relation in equation(4).

One realization of $B_{n}$-braid is as the group of braids on the cylinder, with $g_{0}$ becoming the pure braid in which the first string passes over all the other strings and then around the cylinder. There is a natural 'Young' embedding $B_{n}$-braid $\times$ $B_{m}$-braid $\hookrightarrow B_{n+m}$-braid. One places the second cylinder concentrically inside the first, then allows the two cylinders to converge in such a way that the nodes of $B_{n}$-braid, respectively $B_{m}$-braid, remain consecutive on the edges of the cylinder (while of course preserving over/under information). There is a corresponding embedding $H(n) \times H(m) \hookrightarrow H(n+m)$. The construction of 'standard' modules (in the sense of [11, 58]) follows from this. The quotient $\Psi_{d}$ complicates this, in that the spectrum of $X_{n+1}$ (the image of $\left(1, X_{1}\right)$ ) is not that of $X_{1}$ (the image of $\left(X_{1}, 1\right)$ ).

(3.1) Let $\left\langle X_{i}\right\rangle$ denote the commutative subalgebra $\left\langle X_{i} \mid i=1, \ldots, n\right\rangle \subseteq \mathrm{H}(n, d)$. Generically, as we will see, we may determine a unique basis of primitive (and of course central) idempotents $\epsilon_{x}$ of $\left\langle X_{i}\right\rangle$ with $\sum_{x} \epsilon_{x}=1$. There will be certain specialisations where this basis will not make sense (certain idempotents will have preidempotents with vanishing normalisation). In any case, any primitive idempotent decomposition of 1 in $\mathrm{H}(n, d)$ will be different, but the unique primitive central idempotent decomposition of 1 in $\mathrm{H}(n, d)$ will be expressible as a crudification of the above (albeit depending on $k$ ). The generic case will be the least crude (one idempotent per block/isomorphism class of simples); and it will be necessary, formally, to combine certain of these generic idempotents (into non-simple blocks) to make idempotents which make sense over $k$ in non-semisimple cases. Each $\epsilon_{x}$ must obey $X_{i} \epsilon_{x}=x_{i} \epsilon_{x}$ with $x_{i}$ some scalar. (Thus each induces a left $\mathrm{H}$-module $\Pi_{x}:=\mathrm{H} \epsilon_{x}$. Since the $H_{n}$ subalgebra of $H(n)$ maps isomorphically to its image in the quotient we have $\mathrm{H} \epsilon_{x}=H_{n} \epsilon_{x}$, of rank $n$ !.)

Evidently $x_{1} \in\left\{\lambda_{i}\right\}$, and with $\pi_{j}:=\prod_{i \neq j}\left(X-\lambda_{i}\right)$ we have $X \pi_{j}=\lambda_{j} \pi_{j}$ and $\pi_{j} \epsilon_{x} \propto \epsilon_{x}$. For each such $\pi_{j}$ there exists a minimal polynomial $\pi_{j,-}\left(X_{2}\right)=\prod_{k}\left(X_{2}-\right.$ $\left.\lambda_{j, k}\right)$ such that $\pi_{j} \pi_{j,-}=0$. Set $\pi_{j, k}=\prod_{l \neq k}\left(X_{2}-\lambda_{j, l}\right)$ and $\epsilon_{(j, k)}=\pi_{j} \pi_{j, k}$. Then $X_{2} \epsilon^{*}(j, k)=\lambda_{j, k} \epsilon^{\cdot}(j, k)$. For each $\epsilon^{\cdot}{ }_{(j, k)}$ there exists a polynomial $\pi_{j, k,-}\left(X_{3}\right)$ such that $\pi_{j} \pi_{j, k} \pi_{j, k,-}=0$, and so on. That is, the roots of such a polynomial are certain of the eigenvalues of the $X_{i}$ s.

We can work out these eigenvalues of the $X_{i}$ s by looking at the properties of generically irreducible representations as given in $\$ 1.2$. Let $\mu \in \Lambda_{n}^{d}$. A 'standard' insertion of $\underline{n}=\{1,2, . ., n\}$ into the boxes of $\mu$ is one such that deletion of the boxes containing $\{l, . ., n\}$ produces a legitimate $p$-partition for every $l$. Let $T_{\mu}$ denote the set of all such standard insertions. For $i \in \underline{n}$ and $w \in T_{\mu}$ there is a $k \in\{1,2, . ., d\}$ such that $i$ appears in a box in the $k^{t h}$ partition in $w$. Define $w(i)=k$. It will be evident from the restriction rule (7) that $T_{\mu}$ may be used as a basis for $\Delta_{\mu}$, once equipped with a suitable action.

(3.2) Proposition. The action of the $X_{i}$ s on this basis may be taken to be lower triangular, with diagonal element of $X_{i}$ on $w \in T_{\mu}$ given by $\lambda_{w(i)} q^{2 w^{i}}$ where $w^{i}$ is the distance of $i$ off the main diagonal in the $w(i)^{\text {th }}$ partition in $\mu$ (with distances below the diagonal being negative). 
Proof: In case $n=1$ the claim holds since $R_{i}(X)=\lambda_{i}$. Suppose the claim is true at level $n-1$. Then the eigenvalues for $X_{1}, . ., X_{n-1}$ at level $n$ are given by restriction using the rule (7). The eigenvalues for $X_{n}$ may be determined using the centrality of $\sum_{i} X_{i}$ etc..

(3.3) Let $v_{i}$ denote the $i^{\text {th }}$ elementary vector in $\mathbb{Z}^{d}$. Describe a walk on $\mathbb{Z}^{d}$ (or $\left.\mathbb{Z}^{d} /(1,1, . ., 1)\right)$ by word $w=w_{1} w_{2} \ldots$ in $\underline{d}$ such that the vector between the $i^{t h}$ and $i+1^{\text {st }}$ site visited is $v_{w_{i}}$. For given word $w$ define $\#_{l}(i)=\#_{l}^{w}(i)=\sum_{j=1}^{l} \delta_{w_{j}, i}$. A reflection hyperplane $(i, j ; x)$ is characterised by a pair $v_{i}, v_{j}(i \neq j \in \underline{d})$ not parallel to it, and the signed distance $x$ in the direction of $v_{i}$ of this hyperplane from 0 (i.e. the $x$ such that $0+x v_{i}$ lies on it). A walk touches this hyperplane at $l$ if

$$
\#_{l}(i)-\#_{l}(j)=x
$$

Let $w$ be a walk which touches hyperplane $(i, j ; x)$ at $l$. The walk $w^{\prime}$ got from $w$ by applying permutation $(i j)$ to every $w_{t} t>l$ is called the (affine) reflection of $w$ in $(i, j ; x)$ at $l(\mathrm{NB}$, the touching point $l$ is not in general uniquely defined by $w$ and $(i, j ; x)$; or indeed existent). Every point of the reflection after $l$ is the reflection of this point in $(i, j ; x)$ in the usual alcove geometry sense (see [49, Ch.7]).

If $w^{\prime}$ meets another (not necessarily distinct) hyperplane $(k, m ; y)$ at $l^{\prime}>l$ then of course $w$ meets the image of $(k, m ; y)$ in $(i, j ; x)$ at the same moment. If $w^{\prime \prime}$ is the reflection of $w^{\prime}$ in $(k, m ; y)$ at $l^{\prime}$ then we say $w, w^{\prime}, w^{\prime \prime}$ in the same walk orbit of the reflection group generated by these hyperplanes (the $i^{\text {th }}$ points of these walks are in the same orbit in the usual sense, for each $i$ ). We may think of folding up the space along the set of hyperplanes in the group - the walk orbits are the sets of walks which are mapped into each other by this folding. For $G$ a reflection group generated by hyperplanes we write $w \sim^{G} w^{\prime}$ if walks $w, w^{\prime}$ in the same walk orbit of $G$. Each hyperplane partitions space into two parts (not counting the hyperplane itself). The 'outside' of the hyperplane $(i, j ; x), x \neq 0$, is the part not containing 0 .

We will restrict attention to hyperplanes not touching 0 . Note that for each walk $w$ from 0 which finishes at a point $\mu$ outside some hyperplane there is not in general a unique walk in its orbit which finishes at the image point of $\mu$, inside it. The orbit structure of walks is more complicated than that of points. For example, the endpoints of elements of the same walk orbit are necessarily in the same point orbit; but the converse does not follow. Further, a walk which stays in the interior of the fundamental alcove is in a singleton orbit; and more generally a walk which touches hyperplanes a total of $t$ times has $2^{t}$ elements in its orbit (see figure 11 and figure 2).

For $G$ a reflection group generated by a set of reflection hyperplanes $S$ let $\bar{S} \subset G$ denote the set of simple reflections. It will be convenient to confuse these reflections with the corresponding hyperplanes, noting that $\bar{S} \supset S$ in general. In particular we may now compose hyperplanes by conjugation in $G$, i.e. $(i, j ; x) \circ(j, k ; y)=(i, k ; x+$ $y$ ) and so on. Let $\mathcal{A}^{\prime} \subset \mathcal{A}$ consist of those elements of the form $\left(\lambda_{i}-q^{-2 x} \lambda_{j}\right)$, all $i, j, x$. Define the factor set of $G, \mathcal{F}(G) \subset \mathcal{A}^{\prime}$ such that $(i, j ; x) \in \bar{S}$ iff $\left(\lambda_{i}-q^{-2 x} \lambda_{j}\right) \in \mathcal{F}(G)$. The elements of $\mathcal{F}(G)$ are called factors. Note that there is an equality of ideals

$$
\sum_{(i, j ; x) \in S} \mathcal{A}\left(\lambda_{i}-q^{-2 x} \lambda_{j}\right)=\sum_{(i, j ; x) \in \bar{S}} \mathcal{A}\left(\lambda_{i}-q^{-2 x} \lambda_{j}\right)
$$

since the composition of hyperplanes above corresponds to the addition (up to a unit) of factors. 


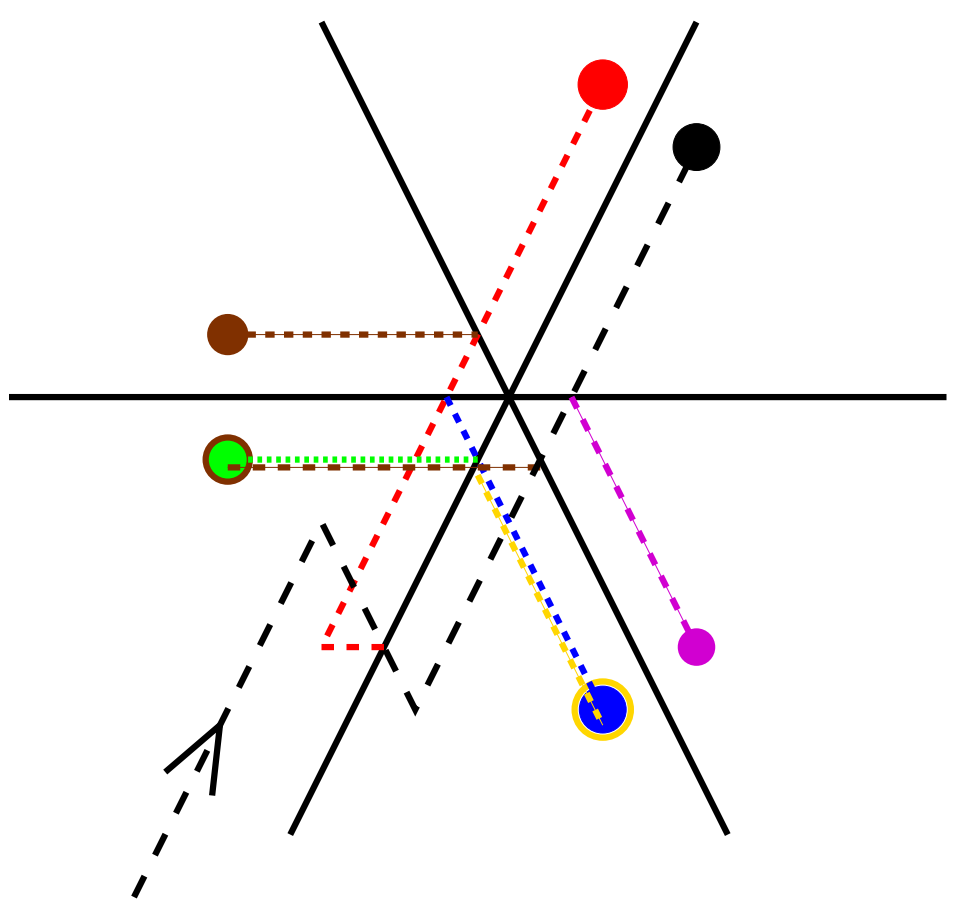

Figure 1: Eight walks in a walk orbit for $d=3$ (origin in the bottom left hand corner, endpoints circled).

For a walk $w$ of length $n$ let $\mu(w)=\left(\#_{n}(1), \#_{n}(2), ..\right)$, an ordered partition of $n$. To each walk $w$ of length $n$ associate an element $\lambda^{w} \in \mathcal{A}^{n}$ as follows:

$$
\left(\lambda^{w}\right)_{i}=\lambda_{w_{i}} q^{2\left(\#_{i}\left(w_{i}\right)-1\right)} .
$$

For example $\lambda^{3331312}=\left(\lambda_{3}, \lambda_{3} q^{2}, \lambda_{3} q^{4}, \lambda_{1}, \lambda_{3} q^{6}, \lambda_{1} q^{2}, \lambda_{2}\right)$.

NB, This $\lambda^{-}$gives an injective map from $\cup_{\mu \in \Lambda^{\mathcal{D}}(n)} T_{\mu}$ into $\mathcal{A}^{n}$.

(3.4) Proposition. (i) Let $w^{\prime}$ be the reflection of $w$ in $(i, j ; x)$ at $l$, then every non-zero element of $\lambda^{w}-\lambda^{w^{\prime}}$ is of the form $\pm q^{2 \alpha}\left(\lambda_{i}-q^{-2 x} \lambda_{j}\right)$ (some $\alpha \in \mathbb{Z}$ ). (NB, no mention of $l$ in the implication.)

(ii) Let $w, w^{\prime}$ be two walks. If every element of $\lambda^{w}-\lambda^{w^{\prime}}$ is divisible by $\left(\lambda_{i}-q^{-2 x} \lambda_{j}\right)$ then $w, w^{\prime}$ are related by one (or more) reflection in $(i, j ; x)$ for some $l$ (resp. $l<$ $\left.l^{\prime}<l^{\prime \prime} \ldots\right)$.

(iii) Let $G$ be the group generated by a set of simple reflections $\{(i, j ; x),(k, l ; y), .$.$\} .$ Walks $w, w^{\prime}$ are in the same walk orbit of $G$ (i.e. related by a sequence of reflections in hyperplanes in $G)$ if and only if each non-zero $\left(\lambda^{w}-\lambda^{w^{\prime}}\right)_{i}$ is divisible by an element of $\mathcal{F}(G)$.

Proof: (i) Consider the first point at which $w$ and $w^{\prime}$ differ, which we may take to be $w_{l+1}^{\prime}=(i j) w_{l+1}=j$. We have $\left(\lambda^{w}-\lambda^{w^{\prime}}\right)_{l+1}=\lambda_{i} q^{2\left(\#_{l+1}^{w}(i)-1\right)}-\lambda_{j} q^{2\left(\#_{l+1}^{w^{\prime}}(j)-1\right)}$. Now $\#_{l+1}^{w}(i)=\#_{l}^{w}(i)+1$ and $\#_{l+1}^{w^{\prime}}(j)=\#_{l}^{w^{\prime}}(j)+1$, so, cf. equation(18), the exponents differ by $2 x$ as required. Since subsequent points $k$ with $w_{k} \neq w_{k}^{\prime}$ are all those at which $\left\{w_{k}, w_{k}^{\prime}\right\} \cap\{i, j\} \neq \emptyset$ the difference in exponents is preserved.

(ii) is a special case of (iii).

(iii) (Only if part) Note that, by construction, $\left(\lambda^{w}-\lambda^{w^{\prime}}\right)_{i}$ is, up to a unit, an element of $\mathcal{A}^{\prime}$. Each pair $w \sim^{G} w^{\prime}$ may be related by a series of simple reflections: 


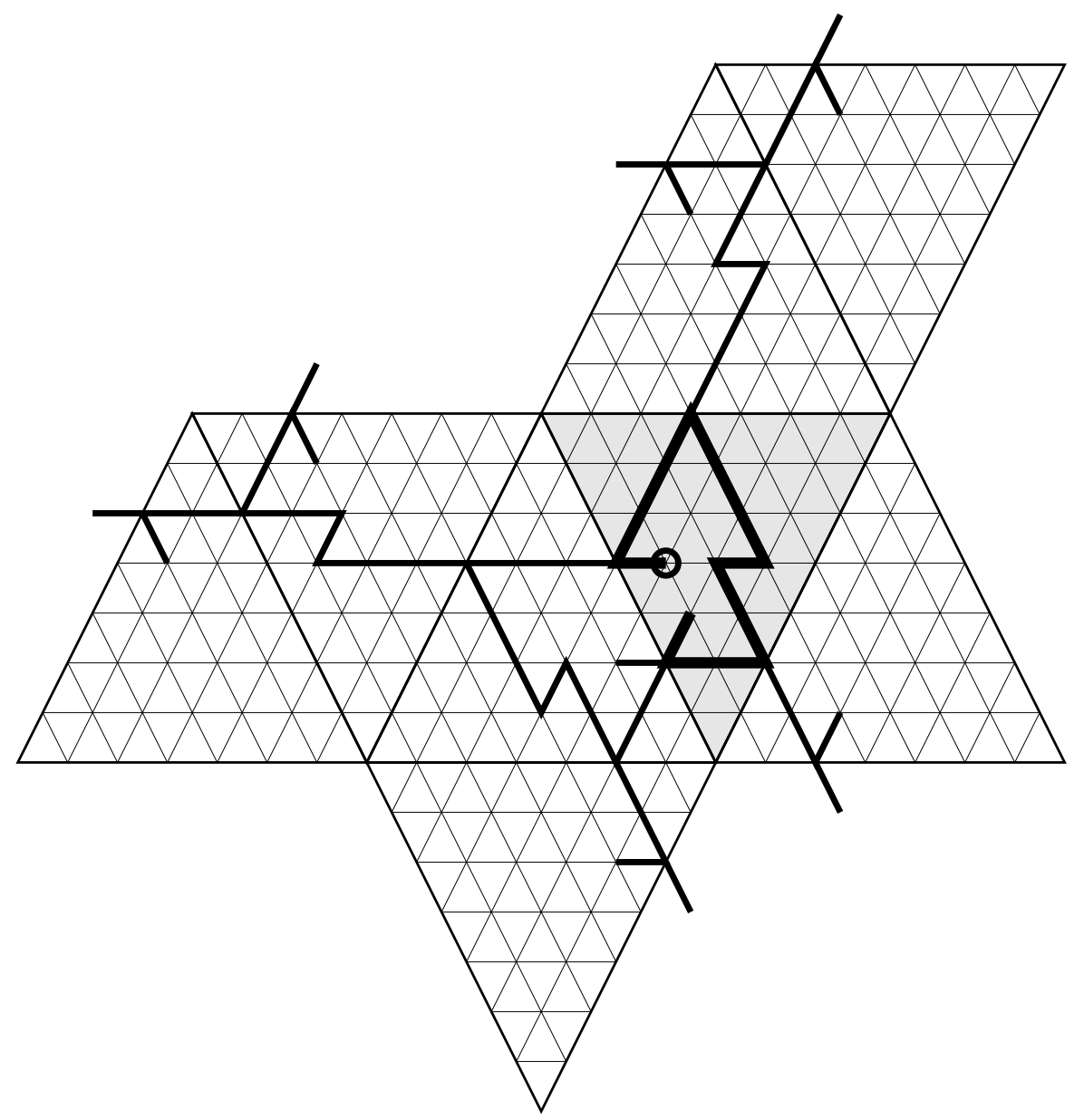

Figure 2: A walk, starting at 0 (circled), in the fundamental alcove (shaded) of a certain $d=3$ affine reflection group; and all walks in its walk orbit. Note that the two endpoints closest to 0 are each reached by two different walks. 
$w \sim^{G} w^{\prime \prime} \sim^{G} . . \sim^{G} w^{\prime}$, and each intermediate $\left(\lambda^{w^{i}}-\lambda^{w^{i+1}}\right)_{i}$ is of the required form by (i). Evidently $\left(\lambda^{w}-\lambda^{w^{\prime}}\right)_{i}=\sum_{i}\left(\lambda^{w^{i}}-\lambda^{w^{i+1}}\right)_{i}$. Now confer equation(19).

(if part) Let $\left.w\right|_{m}$ denote the first $m$ steps in walk $w$. Fix $w$ and consider $w^{\prime}$ such that $w \chi^{G} w^{\prime}$. Suppose that point $m$ is the first at which $\left.\left.w\right|_{m} \chi^{G} w^{\prime}\right|_{m}$. Let $\left.\tilde{w}\right|_{m-1}=$ $\left.w^{\prime}\right|_{m-1}$ and $\tilde{w}_{m}$ be such that $\left.\left.w\right|_{m} \sim^{G} \tilde{w}\right|_{m}$. Thus $\left.\tilde{w}\right|_{m}$ is a reflection of $\left.w^{\prime}\right|_{m}$ in some hyperplane $h$ at $m-1$, with $h \notin G$. Thus $\lambda^{w^{\prime}}-\lambda^{\tilde{w}}=\left(0,0, . ., 0,\left(q^{2 \alpha}\left(\lambda_{i}-q^{-2 x} \lambda_{j}\right)\right)\right)$ for some $\alpha, i, j, x$, where $i \neq j$ since $w^{\prime}$ and $\tilde{w}$ agree at $m-1$. The non-zero factor is not a factor of $\mathcal{F}(G)$ since $h \notin G$. Now consider $\lambda^{w}-\lambda^{w^{\prime}}=\left(\lambda^{w}-\lambda^{\tilde{w}}\right)+\left(\lambda^{\tilde{w}}-\lambda^{w^{\prime}}\right)$. The $m^{\text {th }}$ element of the first summand on the right is divisible by some $f \in \mathcal{F}(G)$ by the only if part, while that of the second summand is not, by the above argument. Thus the LHS is not.

For example, consider the sequences 333,331 and 321 with $\lambda^{333}=\left(\lambda_{3}, q^{2} \lambda_{3}, q^{4} \lambda_{3}\right)$, $\lambda^{331}=\left(\lambda_{3}, q^{2} \lambda_{3}, \lambda_{1}\right)$ and $\lambda^{321}=\left(\lambda_{3}, \lambda_{2}, \lambda_{1}\right)$. We have $(3,1 ; 2) 333=331$ and $(3,2 ; 1) 331=321$ and $\lambda^{333}-\lambda^{321}=\left(0, q^{2}\left(\lambda_{3}-q^{-2} \lambda_{2}\right), q^{4}\left(\lambda_{3}-q^{-4} \lambda_{1}\right)\right)$.

(3.5) Proposition. For each $w \in \cup_{\mu \in \Lambda^{\mathcal{D}}(n)} T_{\mu}$ there is an element $\epsilon_{w}$ of $\left\langle X_{i}\right\rangle$ as described above obeying

$$
X_{i} \epsilon_{w}=\left(\lambda^{w}\right)_{i} \epsilon_{w} .
$$

Proof: For a $d$-partition $\mu$ of form $\left(\left(\mu_{1}\right),\left(\mu_{2}\right), ..\right)$ (i.e. $\left.\mu \in \Lambda^{\mathcal{D}}\right)$ and $w \in T_{\mu}$ identified with the corresponding word $w$ we have $w_{i}=w(i)$ and $\#_{i}\left(w_{i}\right)=w^{i}$. Now cf. proposition 3.2.

Let $R_{x}$ denote the representation of $\left\langle X_{i}\right\rangle$ corresponding to any $\epsilon_{x}{ }_{x}$ as above. It follows that the $\left\langle X_{i}\right\rangle$-simple character of $\operatorname{Res}_{\left\langle X_{i}\right\rangle}^{\mathrm{H}}\left(\Delta_{\nu}\right)$ is

$$
\left[\operatorname{Res}_{\left\langle X_{i}\right\rangle}^{\mathrm{H}}\left(\Delta_{\nu}\right): R_{w}\right]=\delta_{\mu(w), \nu}
$$

$\mathrm{NB}$, this is not a unique characterisation unless:

Cor. If $k$ is generic, i.e. $\lambda^{-}$remains injective on passing from $\mathcal{A}^{n}$ to $(\mathcal{A} \otimes k)^{n}$, then $R_{w} \cong R_{w^{\prime}}$ implies $w=w^{\prime}$ and $\left\langle X_{i}\right\rangle^{\mathcal{D}} \subset H^{\mathcal{D}}$ has enough simples to be semisimple.

(3.6) We define $\mathcal{G}(k)$, the reflection group induced by $k$ as follows. For each triple $(i, j ; x)$ such that $\left(\lambda_{i}-q^{2 x} \lambda_{j}\right) \otimes k=0$ include the hyperplane $(i, j ; x)$ as a generator. Note that this is sensible in as much as $(i, j ; x) \circ(j, k ; y)=(i, k ; x+y)$ while $\lambda_{i}-$ $q^{2 x} \lambda_{j}=0$ and $\lambda_{j}-q^{2 y} \lambda_{k}=0$ imply $\lambda_{i}-q^{2(x+y)} \lambda_{k}=0$. If $d^{\prime}$ of the parameters $\left\{\lambda_{i}\right\}$ are related in this way then the group generated is $S_{d^{\prime}}$, unless $q$ is a root of unity, in which case it is the affine extension. We will usually refer to the group as affine Weyl group regardless, to emphasize the fact that even in the finite case the hyperplanes do not pass through 0 . That is, we exclude from consideration any $k$ in which $\lambda_{i}=\lambda_{j}$ does not imply $i=j$.

Proposition. Over $k, R_{w} \cong R_{w^{\prime}}$ iff $w, w^{\prime}$ are in the same walk orbit of the affine Weyl group induced by $k$ (i.e. related by a some series of reflections).

Proof: There is an isomorphism iff $\lambda^{w}-\lambda^{w^{\prime}}$ vanishes over $k$. Every term is of the form $q^{\alpha}\left(\lambda_{i}-q^{2 x} \lambda_{j}\right)$. Now apply proposition (3.4).

(3.7) Proposition. [Linkage] If there exists a nontrivial homomorphism $\Delta_{\mu} \rightarrow \Delta_{\nu}$ over $k$ then $\mu, \nu$ lie in the same orbit of the affine Weyl group induced by $k$. 
Proof:

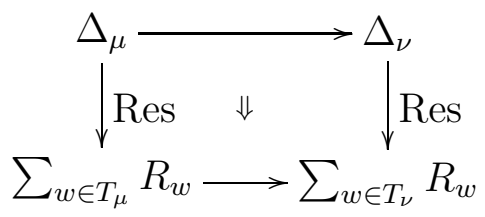

Now apply ((3.6) Proposition).

Note that this strengthens immediately to exclude interorbit maps from any submodule of $\Delta_{\mu}$ to any quotient of $\Delta_{\nu}$, i.e. $\mu \chi^{\mathcal{G}(k)} \nu$ implies $\Delta_{\mu}, \Delta_{\nu}$ have no composition factors in common. Thus, under the assumption that every simple occurs in $\left\{\operatorname{Head}\left(\Delta_{\mu}\right) \mid \mu \in \Lambda^{\mathcal{D}}\right\}$, as for a quasi-hereditary algebra, we have linkage in the form of I6.

(3.8) Proposition. Let $w, w^{\prime} \in \cup_{\mu \in \Lambda^{\mathcal{D}}(n)} T_{\mu}$ be reflections of each other in $(i, j ; x)$ at any $l$. Then over any $k$ in which $\left(\lambda_{i}-q^{2 x} \lambda_{j}\right)$ vanishes we have an isomorphism of left $\mathrm{H}-$ modules

$$
\mathrm{H} \epsilon_{w} \cong \mathrm{H} \epsilon_{w^{\prime}} .
$$

Proof: By ((3.6) Proposition) $\epsilon_{w}$ and $\epsilon_{w^{\prime}}$ induce isomorphic $\left\langle X_{i}\right\rangle$-modules.

(3.9) Given the injectivity of $\lambda^{-}$it will be convenient to be able to refer to a walk $w$ either directly or via its image $\lambda^{w}$. This unifies the labelling schemes for $\epsilon_{w}$ in (3.1) and (3.5).

Note that $R_{x}\left(\pi_{j}\right)=0$ for all $R_{x}$ except those with $x_{1}=\lambda_{j}$. Let $S_{j}$ be the set of possible values of $R_{x}\left(X_{2}\right)$ when $x_{1}=\lambda_{j}$ (i.e. $S_{j}=\left\{\lambda_{k} \neq \lambda_{j}, q^{2} \lambda_{j}, q^{-2} \lambda_{j}\right\}$ ). Then $R_{\left(\lambda_{j}, x_{2}, . .\right)}\left(\prod_{s \in S_{j}}\left(X_{2}-s\right)\right)=0$ for any such $x$, and $\pi_{j} \prod_{s \in S_{j}}\left(X_{2}-s\right)$ lies in the radical of $\left\langle X_{i}\right\rangle$, and hence some power of it vanishes. This tells us, up to multiplicity, the roots of $\pi_{j,-}$, and hence of the preidempotent $\pi_{j} \pi_{j, k}$ (obtained by omitting a factor $\left(X_{2}-\lambda_{j, k}\right), \lambda_{j, k} \in S_{j}$, from $\left.\pi_{j} \pi_{j,-}\right)$. If $\lambda^{-}$is injective then the radical is $\{0\}$ and all the roots can be distinguished. Iterating this argument we have

Proposition. For w a walk

$$
\begin{aligned}
& \epsilon_{w}^{\cdot}=\prod_{i=1}^{|w|} \prod_{x \in \cup_{\nu,|\nu|=i} T_{\nu}}\left(X_{i}-R_{x}\left(X_{i}\right)\right) \\
& \text { such that } \\
& \left.x\right|_{i-1}=\left.w\right|_{i-1} \\
& x_{i} \neq w_{i}
\end{aligned}
$$

where the union is over all multipartitions satisfying the constraints.

For example, $\epsilon^{\cdot}{ }_{111}=\epsilon^{\cdot}{ }_{11}\left(X_{3}-q^{-2} \lambda_{1}\right) \prod_{i=2}^{d}\left(X_{3}-\lambda_{i}\right)$; and

$$
\begin{aligned}
\epsilon_{11122}^{\prime}=\epsilon_{111}^{\prime} & \left(\left(X_{4}-q^{-2} \lambda_{1}\right)\left(X_{4}-q^{6} \lambda_{1}\right) \prod_{2<i \leq d}\left(X_{4}-\lambda_{i}\right)\right) \\
& \cdot\left(\left(X_{5}-q^{-2} \lambda_{1}\right)\left(X_{5}-q^{6} \lambda_{1}\right)\left(X_{5}-q^{-2} \lambda_{2}\right) \prod_{2<i \leq d}\left(X_{5}-\lambda_{i}\right)\right) .
\end{aligned}
$$

Note that unless the omitted factor in $\pi_{j}$ or $\pi_{j, k}$ coincides (under $X_{1} \leftrightarrow X_{2}$ ) with a factor in the other, then the product $\pi_{j} \pi_{j, k}$ is automatically in $Z(\mathrm{H}(2, d))$ (simply 
multiply in all the factors apparently required for symmetry, then replace these with scalars using the eigenvector property - the excluded cases are those where one or more such scalar vanishes).

For example, in case $n=2, d=2\left\langle X_{i}\right\rangle$ has rank 6 . We have $\left(X-\lambda_{2}\right)\left(X_{2}-\right.$ $\left.q^{-2} \lambda_{1}\right)\left(X_{2}-q^{2} \lambda_{1}\right)\left(X_{2}-\lambda_{2}\right)=0$ so, for example, $\epsilon_{11}=\epsilon_{\lambda_{1}, q^{2} \lambda_{1}}=\left(X-\lambda_{2}\right)\left(X_{2}-\right.$ $\left.q^{-2} \lambda_{1}\right)\left(X_{2}-\lambda_{2}\right)$ is a preidempotent with $X_{2} \epsilon_{\lambda_{1}, q^{2} \lambda_{1}}=q^{2} \lambda_{1} \epsilon_{\lambda_{1}, q^{2} \lambda_{1}}$; while $\epsilon_{\lambda_{1} \lambda_{2}}=$ $\left(X-\lambda_{2}\right)\left(X_{2}-q^{-2} \lambda_{1}\right)\left(X_{2}-q^{2} \lambda_{1}\right)$ is a preidempotent with $X_{i} \epsilon_{\lambda_{1} \lambda_{2}}=\lambda_{i} \epsilon_{\lambda_{1} \lambda_{2}}$. The preidempotent $\epsilon_{\lambda_{1}, q^{2} \lambda_{1}}$ lies in $Z(H)$ (provided $q^{2} \neq 1$ ) since it can be symmetrized: $\left(X_{1}-q^{-2} \lambda_{1}\right) \epsilon_{\lambda_{1}, q^{2} \lambda_{1}}=\left(\lambda_{1}-q^{-2} \lambda_{1}\right) \epsilon_{\lambda_{1}, q^{2} \lambda_{1}}$. On the other hand, $\epsilon_{\lambda_{1} \lambda_{2}}$ cannot be rescaled to its symmetrized form because the symmetrizing factor $X_{2}-\lambda_{2}$ kills it.

For $\mathrm{H}(2, d)$ we have $\prod_{j \neq 1}\left(X-\lambda_{j}\right)\left(X_{2}-q^{2} \lambda_{1}\right)\left(X_{2}-q^{-2} \lambda_{1}\right) \prod_{j \neq 1}\left(X_{2}-\lambda_{j}\right)=0$, so similar considerations apply. Indeed they do for all $n$. In particular $\epsilon_{11 . .1} \in Z(\mathrm{H})$ unless $q^{2}=1$. More generally, we may proceed as follows.

(3.10) For $\mu$ an ordered partition of $n$ let $H_{\mu}$ denote the corresponding Young subalgebra of $H_{n} \subset \mathrm{H}(n, d)$. For $w$ a walk let $e_{w} \in H_{n}$ denote the $q$-Young symmetrizer [14] [25, §9.3] associated to $H_{\mu(w)}$. A walk $w$ is said to be sorted if it takes the form 111..22.. (more precisely, if $w_{i} \leq w_{i+1}$ for all consecutive pairs of steps in $w$ ). There is a unique sorted walk in each $T_{\mu}$, denoted $w(\mu)$. A walk $w$ is said to be direct if all steps in a given direction are taken consecutively (thus a sorted walk is direct).

Proposition. For $w$ sorted, and $\left(1+q^{2}\right)$ and each $\lambda_{i}$ invertible, $\epsilon_{w}{ }_{w}$ commutes with $H_{\mu(w)}$.

Proof: Let $\mu^{t}$ denote the $t^{t h}$ interval of $\{1,2, . ., n\}$ in the ordered paritition $\mu(w)$, i.e. the $t^{t h}$ set of integers fixed under the action of the Young subgroup $S_{\mu(w)}$ of $S_{n}$ on $\{1,2, \ldots, n\}$. We require to show, for each $t$, that the factors in $\epsilon^{\cdot}{ }_{w}$ involving $X_{\mu^{t}}:=\left\{X_{i} \mid i \in \mu^{t}\right\}$ constitute a symmetric polynomial in these variables, and hence commute with the $t^{t h}$ factor algebra in $H_{\mu(w)}$ (the remaining factor algebras commute with these variables by equation(15)). Our walk is of the form $w=111 . .22 . . t t .$. , and the factors in question are (by $((\sqrt{3.9})$ proposition $))$ those written out explicitly in:

$$
\begin{gathered}
\epsilon_{w}^{\cdot}=\epsilon_{111 . .22 . .} \prod_{i=t+1}^{d}\left(X_{a}-\lambda_{i}\right)\left(\prod_{i=1}^{t-1}\left(X_{a}-q^{-2} \lambda_{i}\right)\left(X_{a}-q^{2 \mu_{i}(w)} \lambda_{i}\right)\right) \\
\prod_{b \in \mu^{t} \backslash\{a\}}\left(\left(X_{b}-q^{-2} \lambda_{t}\right) \prod_{i=t+1}^{d}\left(X_{b}-\lambda_{i}\right)\left(\prod_{i=1}^{t-1}\left(X_{b}-q^{-2} \lambda_{i}\right)\left(X_{b}-q^{2 \mu_{i}(w)} \lambda_{i}\right)\right)\right) \ldots
\end{gathered}
$$

(where $X_{a}$ is the first $X_{i}$ in $X_{\mu^{t}}$ ). It will be apparent that this is rendered symmetric by multiplying by $\left(X_{a}-q^{-2} \lambda_{t}\right)$, but $\left(X_{a}-q^{-2} \lambda_{t}\right) \epsilon^{\cdot}{ }_{w}=\left(\lambda_{t}-q^{-2} \lambda_{t}\right) \epsilon^{\cdot}$ so it is already symmetric provided that $\left(1-q^{-2}\right) \lambda_{t}$ is invertible.

A similar property holds for direct walks.

(3.11) Proposition. If $w$ takes the form 111..22.. then $\epsilon_{w} e_{w}=e_{w} \epsilon_{w}$ (and similarly for the preidempotent forms $\epsilon$ and $\mathbf{e})$. The modules $\Delta_{\mu(w)}=\mathrm{He}_{w} \epsilon_{w}$ are the left standard modules of $\mathrm{H}$ with these weights.

Proof: Note that $e_{w} \in H_{\mu(w)}$ and apply ((3.10) proposition).

(3.12) Write $\mu \geq \nu$ if every change of direction in $w(\mu)$ occurs at the same step as one in $w(\nu)(\mathrm{NB}, w(\nu)$ may have changes at other points as well). Note that $\mu \geq \nu$ implies $e_{w(\mu)} e_{w(\nu)}=e_{w(\mu)}$, and hence $\mathbf{e}_{w(\mu)} \mathbf{e}_{w(\nu)}=\kappa_{\nu} \mathbf{e}_{w(\mu)}$ for some $\kappa_{\nu} \in \mathcal{A}$.

Proposition. Suppose that $w(\mu) \sim^{\mathcal{G}(k)} w(\nu)$, and $\mu \geq \nu$, and $\kappa_{\nu} \otimes k \neq 0$. Then $\Delta_{\mu} \hookrightarrow \Delta_{\nu}$. 
Proof:

$$
\Delta_{\mu}=\mathrm{He}_{w(\mu)} \epsilon_{w(\mu)}^{*}=\mathrm{He}_{w(\mu)} \mathbf{e}_{w(\nu)} \epsilon_{w(\mu)}^{\cdot} \hookrightarrow \mathrm{He}_{w(\nu)} \epsilon^{*}{ }_{w(\mu)} \cong \mathrm{He}_{w(\nu)} \epsilon_{w(\nu)}^{\prime}=\Delta_{\nu} .
$$

\section{The case $d=2$ and the blob algebra}

\subsection{Idempotents in $\mathbf{H}(n, 2)$}

The primitive and central idempotents $e_{n}^{ \pm l}$ corresponding to the four one-dimensional representations of $\mathrm{H}(n, 2)$ over $\mathcal{K}$ may be constructed as follows.

Fixing $d=2$ define

$$
P_{n}=P_{n}^{+2}=q^{n-1}[n]\left(q^{2 n-2} \lambda_{2}-\lambda_{1}\right)
$$

and $P_{n}^{-2}=t P_{n}, P_{n}^{+1}=s P_{n}$ and $P_{n}^{-1}=s t P_{n}$.

(4.1) Proposition. Set

$$
\alpha_{j}=\frac{-\lambda_{1}}{P_{j}} \quad \beta_{j}=\frac{q P_{j-1}}{P_{j}} \quad \gamma_{j}=\frac{q^{2 j-2}}{P_{j}} .
$$

Then $e_{0}^{+2}=1$ and

$$
e_{j+1}^{+2}=e_{j}^{+2}\left(\alpha_{j+1}+\beta_{j+1} g_{j}+\gamma_{j+1} X_{j+1}\right) e_{j}^{+2}
$$

and $e_{j}^{-2}=t e_{j}^{+2}, e_{j}^{+1}=s e_{j}^{+2}$, and $e_{j}^{-1}=t s e_{j}^{+2}$.

Proof: see $\oint$ 5.1 (or simply consider $g_{j-1} e_{j}^{+2}$ ).

Examples:

$$
\begin{gathered}
e_{1}^{-2}=e_{1}^{+2}=\frac{X_{1}-\lambda_{1}}{\lambda_{2}-\lambda_{1}} \\
e_{2}^{-2}=\left(\frac{X-\lambda_{1}}{\lambda_{2}-\lambda_{1}}\right) \frac{-\lambda_{1}+q^{-2} g_{1} X g_{1}-q^{-1}\left(\lambda_{2}-\lambda_{1}\right) g_{1}}{\left(1+q^{-2}\right)\left(q^{-2} \lambda_{2}-\lambda_{1}\right)}\left(\frac{X-\lambda_{1}}{\lambda_{2}-\lambda_{1}}\right) .
\end{gathered}
$$

Define

$$
\Sigma=\left(X_{1}+X_{2}-\left(\lambda_{1}+\lambda_{2}\right)\right) \quad \Pi=\left(X_{1} X_{2}-\lambda_{1} \lambda_{2}\right) .
$$

$\mathrm{NB}, \mathfrak{D}_{2}^{2}=\left\{1, \Sigma, \Pi, \Sigma g_{1}, \Pi g_{1}\right\}$ is a basis of $Z(\mathrm{H}(2,2))$ (see appendix, eqn.(56)).

In terms of $\mathfrak{C}_{2}^{2}$ and $\mathfrak{D}_{2}^{2}$ we have

$$
\begin{gathered}
e_{2}^{-2}=\frac{\lambda_{1}^{2}-\lambda_{1}\left(X+X_{2}\right)+X X_{2}+q^{-1} \lambda_{1}\left(X+X_{2}-\left(\lambda_{1}+\lambda_{2}\right)\right) g_{1}-q^{-1}\left(X X_{2}-\lambda_{1} \lambda_{2}\right) g_{1}}{\left(\lambda_{1}-\lambda_{2}\right)\left(1+q^{-2}\right)\left(q^{-2} \lambda_{2}-\lambda_{1}\right)} \\
=\frac{\left(-\lambda_{1} \Sigma+\Pi\right)\left(1-q^{-1} g_{1}\right)}{\left(\lambda_{2}-\lambda_{1}\right)\left(q^{-2} \lambda_{2}-\lambda_{1}\right)\left(1+q^{-2}\right)}
\end{gathered}
$$

which form manifests the centrality of this idempotent. Note that the preidempotent

$$
\mathbf{e}_{2}^{-2}=\left(-\lambda_{1} \Sigma+\Pi\right)\left(1-q^{-1} g_{1}\right)
$$

coincides with its $s$-image (up to a unit in $\mathcal{A}$ ) if and only if $\lambda_{1}=\lambda_{2}$ in $k$. However,

$$
e_{2}^{-2}+e_{2}^{-1}=\frac{q^{-2} \Sigma+\left(1+q^{-2}\right) \Pi}{\left(q^{-2} \lambda_{2}-\lambda_{1}\right)\left(q^{-2} \lambda_{1}-\lambda_{2}\right)} \frac{\left(q-g_{1}\right)}{[2]} .
$$


A remark is in order on denominators and idempotent decompositions of 1 . The idempotent decomposition of $1=e_{1}^{+2}+e_{1}^{+1} \in \mathrm{H}(1,2)$ is not defined in case $\lambda_{1}=\lambda_{2}$ over $k$, and the radical $\operatorname{rad}(\mathrm{H}(1,2))=k \mathbf{e}_{1}^{+1}$. Obviously $R_{ \pm 1}=R_{ \pm 2}$ for any $n$ in this case. The decomposition of $1=\left(e_{1}^{+2}+e_{1}^{+1}\right)+e_{((1),(1))}+\left(e_{1}^{-2}+e_{1}^{-1}\right) \in \mathrm{H}(2,2)$ thus has the same limitation — the bracketed sums do not split. Similarly when $q=-q^{-1}$ we have $R_{+i}=R_{-i}$ (any $n$ ). More interesting is the case $\lambda_{2}=q^{2} \lambda_{1}$. Here both $e_{2}^{-2}$ and $e_{2}^{+1}$ are undefined. Clearly $R_{+1} \neq R_{-2}$ (unless $q^{2}=-1, \lambda_{i}=0$ ) so $e_{2}^{-2}+e_{2}^{+1}$ is also undefined, but $e_{2}^{+2}$ and $e_{2}^{-1}$ are well-defined so $e_{2}^{-2}+e_{2}^{+1}+e_{((1),(1))}$ does not split and $R_{+1}, R_{-2}$ (i.e. $\Delta_{((2),)}$ and $\left.\Delta_{\left(,\left(1^{2}\right)\right)}\right)$ both must be composition factors of $\Delta_{((1),(1))}$. NB, at first site this seems problematic for our proposed $H^{\mathcal{D}}$ weight space, however over this $k$ we may identify $R_{-2}$ with the simple head of $\Delta_{((1),(1))}$ and label it accordingly (rather than by its $\mathrm{H}(n, d)$ label, which is $\left(,\left(1^{2}\right)\right)$ ). This is a good paradigm for the subtleties with labels in realising ingredient 2(ii) (cf. [56]). In terms of the $A_{1}$ integral weight set $\mathbb{Z} \subset \mathbb{R}$ (recall $\mathfrak{P}^{2}((,(2)))=-2$, $\left.\mathfrak{P}^{2}(((1),(1)))=0, \mathfrak{P}^{2}(((2)))=2,\right)$ we depict the standard modules and morphism by:

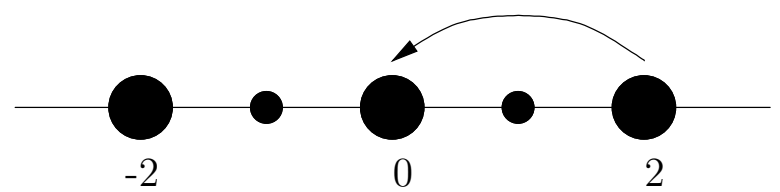

(4.2) It will be convenient to note the equality

$$
\left(X-\lambda_{2}\right)\left(-\lambda_{1} \Sigma+\Pi\right)=0
$$

and its $s$ image; and hence that

$$
\left(-\lambda_{1} \Sigma+\Pi\right) e_{2}^{-2}=\left(-\lambda_{1} \Sigma+\Pi\right) \frac{g_{1}-q}{-q^{-1}-q}
$$

and $s$ image.

(4.3) Now consider the algebras $H^{\mathcal{D}}(n, 2)$ obtained by quotienting by $\mathbf{e}_{2}^{-1}=0$, $\mathbf{e}_{2}^{-2}=0$.

First consider $n=2$. A basis of the ideal $\mathcal{D}_{2}(2)$ is simply $\left\{\mathbf{e}_{2}^{-1}, \mathbf{e}_{2}^{-2}\right\}\left(\lambda_{1} \neq \lambda_{2}\right)$. The basis $\mathfrak{C}_{2}^{2}$ of $\mathrm{H}(2,2)$ is

$$
\left\{1, X_{1}, X_{2}, X_{1} X_{2}, g_{1}, X_{1} g_{1}, X_{2} g_{1}, X_{1} X_{2} g_{1}\right\} .
$$

We can think of using the relation $e_{2}^{-2}=0$ to eliminate $X_{1} X_{2} g_{1}$, and then using its $s$-partner to eliminate $g_{1}$, to obtain a basis for $H^{\mathcal{D}}(2,2)$ (see also $\S 5.3$ ). Specifically, it is convenient to represent the quotient relations as

$$
\left(X_{1}+X_{2}-\left(\lambda_{1}+\lambda_{2}\right)\right)\left(g_{1}-q\right)=0 \quad\left(X_{1} X_{2}-\lambda_{1} \lambda_{2}\right)\left(g_{1}-q\right)=0 .
$$

N.B. These two relations generate the same ideal.

Alternatively, rewriting $g_{i}-q=: u_{i}\left(\right.$ so $\left.u_{i}^{2}=-\left(q+q^{-1}\right) u_{i}\right)$ and $X-\lambda_{1}=: v$ we have

$$
v u_{1} v u_{1}=\left(\lambda_{1} q^{-1}-\lambda_{2} q\right) v u_{1} \quad u_{1} v u_{1} v=\left(\lambda_{1} q^{-1}-\lambda_{2} q\right) u_{1} v
$$

and similarly for the image under $s$. Combining (or by applying $\left(g_{1}-q\right)$ to (27i) from the left) we obtain

$$
u_{1} v u_{1}=\left(\lambda_{1} q^{-1}-\lambda_{2} q\right) u_{1}
$$

(and $s$ image). 


\section{2 $H^{\mathcal{D}}(n, 2)$ and the blob algebra}

The blob algebra $b_{n}$ [52] may be defined by generators $\left\{e_{-}, U_{1}, U_{2}, \ldots, U_{n-1}\right\}$ and relations $e_{-} e_{-}=e_{-}, U_{i} U_{i}=-[2] U_{i}$,

$$
\begin{gathered}
U_{i} U_{i \pm 1} U_{i}=U_{i} \\
U_{1} e_{-} U_{1}=y_{e} U_{1},
\end{gathered}
$$

generators commute otherwise, and $q, y_{e}$ are parameters. (1) (The ordinary TemperleyLieb algebra $T_{n}(q)$ is the subalgebra generated by the $U_{i}$ s.) The properties $11-6$ are established for the blob algebra in [54].

There is a 'good' parameterisation of $b_{n}$ by $q$ and $m$, where $y_{e}=-\frac{[m-1]}{[m]}$. In this parameterisation it is convenient to replace $e_{-}$by the rescaled generator $U_{0}=$ $-[m] e_{-}$, or rather to replace $e_{-}$with $U_{0}$, with relations $U_{0}^{2}=-[m] U_{0}$ and

$$
U_{1} U_{0} U_{1}=[m-1] U_{1}
$$

The variant with these relations is isomorphic to the original except (obviously) when $[m]=0$.

(4.4) Proposition. Let $m, q, \lambda_{1}, \lambda_{2}$ be such that

$$
[m-1]\left(\lambda_{1}-\lambda_{2}\right)=\left(\lambda_{1} q^{-1}-\lambda_{2} q\right)[m]
$$

$\left(N B, \lambda_{1} \neq \lambda_{2}\right.$ unless $q^{2}=1$ and $m \rightarrow \infty$, or $[m]=0$; otherwise, putting $\frac{\lambda_{1}}{\lambda_{2}}=q^{-2 r}$ then $m=r$ - we may take $\lambda_{1}=\frac{q^{m}}{q-q^{-1}}$, then $\left.\lambda_{1}-\lambda_{2}=[m]\right)$. Then there is an isomorphism

$$
\phi: H^{\mathcal{D}}(n, 2) \rightarrow b_{n}
$$

given by $\phi\left(u_{i}\right)=U_{i}$ and $\phi(v)=U_{0}$. There is a homomorphism $\psi: b_{n} \rightarrow H^{\mathcal{D}}(n, 2)$ given by $\psi\left(U_{i}\right)=u_{i}, \psi\left(U_{0}\right)=v$.

Proof: It is straightforward to verify the cyclotomic Hecke relations under $\phi$. A direct calculation also shows that the image under $\phi$ of the numerator of $e_{2}^{-2}$, as in equation (23), vanishes identically if we use the form of $\lambda_{1}$ given in the proposition. The image of $e_{2}^{-1}$ vanishes similarly. Thus $\phi$ is a surjective homomorphism (except possibly when $q^{2}=1$ ). A dimension count reveals that this surjective map is generically an isomorphism.

For $\psi$, equation(29) verifies relation(32). It is now enough to check $u_{1} u_{2} u_{1}=u_{1}$ (i.e., in $\left.\mathrm{H}(3,2), u_{1} u_{2} u_{1}-u_{1}=u_{1}\left(u_{2} u_{1}-1\right)=[3] ! e_{3}^{-} \in \mathcal{D}_{2}\right)$. Write $f=u_{1} u_{2} u_{1}-u_{1}$ and consider $f \mathcal{D}_{2} f$ (if $f \in \mathcal{D}_{2}$ then $f \in f \mathcal{D}_{2} f$, at least if [3]! is invertible, and this is a much smaller and simpler object to work with). This (pre)idempotent subalgebra includes

$$
\begin{gathered}
f\left(X_{1}+X_{2}-\left(\lambda_{1}+\lambda_{2}\right)\right) f=f\left(\left(1+q^{-2}\right) X-\left(\lambda_{1}+\lambda_{2}\right)\right) f \\
f\left(X_{1} X_{2}-\lambda_{1} \lambda_{2}\right) f=f\left(-q^{-1} X g_{1} X-\lambda_{1} \lambda_{2}\right) f
\end{gathered}
$$

and

$$
f X g_{1} g_{2}\left(X_{1}+X_{2}-\left(\lambda_{1}+\lambda_{2}\right)\right) f=f\left(X g_{1} g_{2} X+X g_{1} g_{2} g_{1} X g_{1}-X g_{1} g_{2}\left(\lambda_{1}+\lambda_{2}\right)\right) f
$$

\footnotetext{
${ }^{5}$ The blob algebra is usually defined in terms of a basis of decorated Temperley-Lieb diagrams (hence its name). That the presentation here is equivalent may be verified by a straightforward (if tedious) generalisation of the corresponding exercise for the ordinary Temperley-Lieb algebra as in $[49]$.
} 
$=f\left(-q^{-1} X g_{1} X+X g_{2} g_{1} g_{2} X g_{1}-q^{-2}\left(\lambda_{1}+\lambda_{2}\right) X\right) f=f\left(-\left(q^{-1}+q^{-3}\right) X g_{1} X-q^{-2}\left(\lambda_{1}+\lambda_{2}\right) X\right) f$

A linear combination of these is

$$
[3] !\left(\left(1+q^{-4}\right) \lambda_{1} \lambda_{2}-q^{-2}\left(\lambda_{1}^{2}+\lambda_{2}^{2}\right)\right) f
$$

so $f \in f \mathcal{D}_{2} f$ at least in an open subset of parameter space. The coefficient may be rewritten [3]! $q^{-2} \lambda_{1} \lambda_{2}\left(q-q^{-1}\right)^{2}[m+1][m-1]$ so this covers most interesting cases. The remainder require a more tedious calculation.

Corollary. The finite characteristic $b_{n}$ decomposition matrices in 15] are a subset of type-B Hecke decomposition matrices via the correspondence in $\$ 1.9$.

(4.5) Recall that $m= \pm 1$ implies the existence of a quotient algebra isomorphic to the Temperley-Lieb algebra (in addition to the noted T-L subalgebra). The quotient identifies $X=1$ (or $\left.q^{2}\right)$.

\section{On general $d$}

\subsection{Idempotents in $\mathbf{H}(n, d)$}

The primitive and central idempotents corresponding to the $2 d$ one-dimensional representations of $\mathrm{H}(n, d)$ over $\mathcal{K}$ may be constructed as follows. Fixing $d$, define

$$
P_{n}=P_{n}^{+1}=q^{n-1}[n] \prod_{i \neq 1}\left(q^{2 n-2} \lambda_{1}-\lambda_{i}\right)
$$

$P_{n}^{-1}=t P_{n}, P_{n}^{+2}=s P_{n}, P_{n}^{+3}=s^{2} P_{n}$ and so on. Define $\lambda^{(0)}=1$ and

$$
\lambda^{(i)}=\sum_{d \geq j_{1}>j_{2}>\cdots>j_{i}>1}\left(\prod_{l=1}^{i}-\lambda_{j_{l}}\right)
$$

(sum over descending positive integer sequences $\left(j_{1}, j_{2}, \ldots, j_{i}\right)$, with $j_{1} \leq d$ ).

(5.1) Proposition. Set

$$
\begin{gathered}
\beta_{j+1}=\frac{q P_{j}}{P_{j+1}} \\
\alpha_{j}^{0}=\frac{\prod_{i \neq 1}\left(-\lambda_{i}\right)}{P_{j}} \quad \alpha_{j+1}^{d-i}=\frac{q^{2 j} \lambda^{(i-1)}+\sum_{l=2}^{i} q^{(i-1)(2 j-2)}\left(q^{2 j}-1\right) \lambda_{1}^{l-1} \lambda^{(i-l)}}{P_{j+1}} \quad(1 \leq i<d) .
\end{gathered}
$$

Then $e_{0}^{+1}=1$

$$
e_{j+1}^{+1}=e_{j}^{+1}\left(\beta_{j+1} g_{j}+\sum_{i=0}^{d-1} \alpha_{j+1}^{i} X_{j+1}^{i}\right) e_{j}^{+1}
$$

$e_{j}^{-1}=t e_{j}^{+1}$, and so on .

For example $\beta_{1}=0, \alpha_{1}^{i}=\lambda^{(d-1-i)}$, and

$$
e_{1}^{+1}=\frac{\prod_{i \neq 1}\left(X_{1}-\lambda_{i}\right)}{P_{1}} .
$$

(There are more examples in $\$ 5.2$.)

Proof: 
We work by induction on $j$, with the example above as base. Firstly note that $e_{j+1}^{+1} e_{j}^{+1}=e_{j}^{+1} e_{j+1}^{+1}=e_{j+1}^{+1}$ so, from proposition 2.7, equation(36) gives a correct form for the $R_{+1}$ idempotent up to coefficients. Then note that in this form it is sufficient, cf. equation ( 8$)$, to check the identity $\left(g_{j}-q\right) e_{j+1}^{+1}=0$, and normalisation (i.e. idempotency). The former is a direct calculation, and the later may be checked by evaluating in $R_{+1}$, i.e. substituting $q$ for $g_{i}$ and $\lambda_{1}$ for $X$ (in which case $e_{j+1}^{+1}$ must evaluate to 1$)$. To begin we rewrite the claimed expression for $\left(g_{j}-q\right) e_{j+1}^{+1}$ as

$$
\left(g_{j}-q\right)\left(e_{j}^{+1}\left(\beta_{j+1} g_{j}\right) e_{j}^{+1}+\left(\sum_{i=0}^{d-1} \alpha_{j+1}^{i} X_{j+1}^{i}\right) e_{j}^{+1}\right)
$$

using commutation properties. By the inductive assumption this is

$$
\begin{gathered}
=\left(g_{j}-q\right)\left(e_{j-1}^{+1}\left(\beta_{j} g_{j-1}+\sum_{i=0}^{d-1} \alpha_{j}^{i} X_{j}^{i}\right)\left(\beta_{j+1} g_{j}\right) e_{j}^{+1}+\left(\sum_{i=0}^{d-1} \alpha_{j+1}^{i} X_{j+1}^{i}\right) e_{j}^{+1}\right) \\
=e_{j-1}^{+1} \beta_{j+1}\left(\beta_{j}\left(g_{j}-q\right) g_{j-1} g_{j}+\left(g_{j}-q\right)\left(\sum_{i=0}^{d-1} \alpha_{j}^{i} X_{j}^{i}\right) g_{j}\right) e_{j}^{+1}+\left(g_{j}-q\right)\left(\sum_{i=0}^{d-1} \alpha_{j+1}^{i} X_{j+1}^{i}\right) e_{j}^{+1} \\
=e_{j-1}^{+1} \beta_{j+1}\left(\left(g_{j}-q\right)\left(\sum_{i=0}^{d-1} \alpha_{j}^{i} X_{j}^{i}\right) g_{j}\right) e_{j}^{+1}+\left(g_{j}-q\right)\left(\sum_{i=0}^{d-1} \alpha_{j+1}^{i} X_{j+1}^{i}\right) e_{j}^{+1}
\end{gathered}
$$

Now use the commutation rules on the first summand to bring the factor $g_{j}$ forward through the $X_{j}^{i} \mathrm{~s}$ :

$=\left(g_{j}-q\right)\left(\left(g_{j} \beta_{j+1} \alpha_{j}^{0}+\alpha_{j+1}^{0}\right)+\sum_{i=1}^{d-1}\left(\beta_{j+1}\left(-q \alpha_{j}^{i}-\left(q-q^{-1}\right)\left(\sum_{l=i+1}^{d-1} \alpha_{j}^{l}\left(X_{j}\right)^{l-i}\right)\right)+\alpha_{j+1}^{i}\right) X_{j+1}^{i}\right) e_{j}^{+1}$

but $X_{j} e_{j}^{+1}=q^{2 j-2} \lambda_{1} e_{j}^{+1}$ so we have

$=\left(g_{j}-q\right)\left(\left(-q^{-1} \beta_{j+1} \alpha_{j}^{0}+\alpha_{j+1}^{0}\right)+\right.$

$$
\left.\sum_{i=1}^{d-1}\left(\beta_{j+1}\left(-q \alpha_{j}^{i}-\left(q-q^{-1}\right)\left(\sum_{l=i+1}^{d-1} \alpha_{j}^{l}\left(q^{2 j-2} \lambda_{1}\right)^{l-i}\right)\right)+\alpha_{j+1}^{i}\right) X_{j+1}^{i}\right) e_{j}^{+1}
$$

and equating coefficients to zero we get

$$
\begin{gathered}
\alpha_{j+1}^{0}=q^{-1} \alpha_{j}^{0} \beta_{j+1} \\
\alpha_{j+1}^{d-1}=q \alpha_{j}^{d-1} \beta_{j+1} \\
\alpha_{j+1}^{d-2}=\left(q \alpha_{j}^{d-2}+\left(q-q^{-1}\right) \alpha_{j}^{d-1}\left(q^{2 j-2} \lambda_{1}\right)\right) \beta_{j+1}
\end{gathered}
$$

and

$$
\alpha_{j+1}^{i}=\left(q \alpha_{j}^{i}+\left(q-q^{-1}\right) \sum_{l=i+1}^{d-1} \alpha_{j}^{l}\left(q^{2 j-2} \lambda_{1}\right)^{l-i}\right) \beta_{j+1} \quad(0<i<d) .
$$

Without regard for normalization, any one coefficient may be chosen arbitrarily, so without loss of generality try $\beta_{j+1}=\frac{q P_{j}}{P_{j+1}}$. Then $\alpha_{j+1}^{d-1}=\frac{\rho_{j+1}^{d-1}}{P_{j+1}}$ where $\rho_{j+1}^{d-1}=q^{2 j}, \alpha_{j+1}^{0}=\frac{\lambda^{(d-1)}}{P_{j+1}}$, and

$$
\alpha_{j+1}^{d-2}=\frac{\rho_{j+1}^{d-2} \lambda_{1}+\rho_{j+1}^{d-1} \lambda^{(1)}}{P_{j+1}}
$$

for some $\rho_{j+1}^{d-2}$. Then

$$
\rho_{j+1}^{d-2}=q^{2} \rho_{j}^{d-2}+\left(q^{2}-1\right) q^{2 j-2} q^{2 j-2}=q^{2 j-2}\left(q^{2 j}-1\right) .
$$


Similarly

$$
\alpha_{j+1}^{d-3}=\frac{\rho_{j+1}^{d-3} \lambda_{1}^{2}+\rho_{j+1}^{d-2} \lambda^{(1)} \lambda_{1}+\rho_{j+1}^{d-1} \lambda^{(2)}}{P_{j+1}}
$$

and for $1 \leq i<d$

$$
\alpha_{j+1}^{d-i}=\frac{\sum_{l=1}^{i} \rho_{j+1}^{d-l} \lambda_{1}^{l-1} \lambda^{(i-l)}}{P_{j+1}}
$$

for some $\rho_{j+1}^{-}$. Then

$$
\begin{gathered}
\rho_{j+1}^{d-3}=q^{2} \rho_{j}^{d-3}+\left(q^{2}-1\right)\left(q^{2 j-2} q^{2 j-4}\left(q^{2 j-2}-1\right)+\left(q^{2 j-2}\right)^{3}\right)=q^{4 j-4}\left(q^{2 j}-1\right) \\
\rho_{j+1}^{d-4}=q^{2} \rho_{j}^{d-4}+\left(q^{2}-1\right)\left(\left(q^{2 j-2}\right) q^{4 j-8}\left(q^{2 j-2}-1\right)+\left(q^{2 j-2}\right)^{2} q^{2 j-4}\left(q^{2 j-2}-1\right)+\left(q^{2 j-2}\right)^{4}\right)=q^{6 j-6}\left(q^{2 j}-1\right)
\end{gathered}
$$

and

$$
\rho_{j+1}^{d-i}=q^{(i-1)(2 j-2)}\left(q^{2 j}-1\right) \quad(1<i<d) .
$$

Finally, the normalization condition,

$$
\lambda^{(d-1)}+\sum_{i=1}^{d-1}\left(\left(q^{2 j} \lambda^{(i-1)}+\sum_{l=2}^{i} q^{(l-1)(2 j-2)}\left(q^{2 j}-1\right) \lambda_{1}^{l-1} \lambda^{(i-l)}\right)\left(q^{2 j} \lambda_{1}\right)^{d-i}\right)=P_{j+1}-q^{2} P_{j}
$$

is verified by direct computation.

By inspection of these idempotents we see that the algebras will not be generic in specialisations in which $\frac{\lambda_{i}}{\lambda_{j}}=q^{2 r}$ for some $i, j, r \in \mathbb{N}$; and in certain specialisations in which $q$ is a root of unity (this also follows immediately from [1, [56]). Let us disregard, for the moment, the cases in which $X$ is degenerate or non-invertible. Then noting that rescaling all the $\lambda_{i}$ s by the same factor produces an isomorphic algebra we can fix $\lambda_{1}=1$ (say) and adopt as parameters $q, \frac{\lambda_{i}}{\lambda_{1}}$ [56]. It is illuminating to proceed by example (and cf. [55, §5]).

\subsection{The case $d=3$}

The primitive and central idempotents corresponding to the six one-dimensional representations of $\mathrm{H}(n, 3)$ may be constructed as follows. Fixing $d=3$, define $P_{n}=P_{n}^{+1}$ and $P_{n}^{ \pm i}$ as in equation (33). For example $P_{1}=P_{1}^{ \pm 1}=\left(\lambda_{1}-\lambda_{2}\right)\left(\lambda_{1}-\lambda_{3}\right)$, $P_{2}=q[2]\left(q^{2} \lambda_{1}-\lambda_{2}\right)\left(q^{2} \lambda_{1}-\lambda_{3}\right)$.

As before $\beta_{j}=\frac{q P_{j-1}}{P_{j}}$, and here

$$
\alpha_{j}^{0}=\frac{\prod_{i \neq 1}\left(-\lambda_{i}\right)}{P_{j}} \quad \alpha_{j}^{1}=\frac{q^{2 j-4}\left(q^{2 j-2}-1\right) \lambda_{1}-q^{2 j-2}\left(\lambda_{2}+\lambda_{3}\right)}{P_{j}} \quad \alpha_{j}^{2}=\frac{q^{2 j-2}}{P_{j}}
$$

Compute $e_{j}^{ \pm l}$ by $e_{0}^{+1}=1$ and then

$$
e_{j+1}^{+1}=e_{j}^{+1}\left(\beta_{j+1} g_{j}+\alpha_{j+1}^{0}+\alpha_{j+1}^{1} X_{j+1}+\alpha_{j+1}^{2} X_{j+1}^{2}\right) e_{j}^{+1}
$$

and $e_{j}^{-1}=t e_{j}^{+1}$, and so on. For example

$$
e_{1}^{+1}=\frac{X_{1}^{2}-\left(\lambda_{2}+\lambda_{3}\right) X_{1}+\lambda_{2} \lambda_{3}}{P_{1}}
$$

and

$$
e_{2}^{+1}=e_{1}^{+1}\left(\frac{q P_{1} g_{1}+\lambda_{2} \lambda_{3}+\left(\left(q^{2}-1\right) \lambda_{1}-q^{2}\left(\lambda_{2}+\lambda_{3}\right)\right) X_{2}+q^{2} X_{2}^{2}}{P_{2}}\right) e_{1}^{+1}
$$




$$
=q \frac{\left(X_{1}^{2}-\left(\lambda_{2}+\lambda_{3}\right) X_{1}+\lambda_{2} \lambda_{3}\right)}{P_{1}} \frac{\left(X_{2}^{2}-\left(\lambda_{2}+\lambda_{3}\right) X_{2}+\lambda_{2} \lambda_{3}\right)\left(g_{1}+q^{-1}\right)}{P_{2}}
$$

Given these results, let us consider the generalised Soergel procedure for $d=3$ corresponding to that in $\S 1.1$ for $d=2$. For illustration we consider a field $k$ in which the equations $\frac{\lambda_{i}}{\lambda_{1}}=q^{-n_{i}}(i=2,3)$ and $\frac{\lambda_{1}}{\lambda_{3}}=q^{-n_{1}}$ are solved for positive integer $n_{i}$ only in case $n_{2}=2, n_{3}=4$ (in particular, in this instance $q$ is not a root of unity). Figure 3 illustrates the location of the corresponding reflection hyperplanes (shown as thick lines) in the $H^{\mathcal{D}}$, i.e. $A_{2}$, weight space. The Kazhdan-Lusztig polynomials for this geometry are given in the figure at [55, p.1289]. The claim, then, is that if $\mu$ is a weight in the fundamental alcove $A^{0}$ (such as 0 ) then $\Delta(\mu)$ has simple content (and Loewy structure)

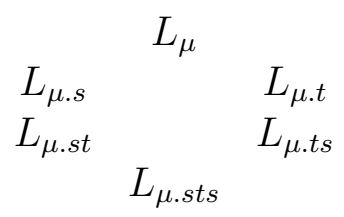

(here $s$ and $t$ are the walls of $A^{0}$ - we are abusing the notation A.s of $\S 1.1$ to apply to weights in the obvious way). Of course, localising at small $n$, some of these simple modules will vanish. Let us consider $n=0,1,2,3$.

(For any $k$ ) we have $H^{\mathcal{D}}(0,3) \cong k$, with basis $\{1\}$. The weight for the corresponding simple module is the innermost dot in the figures (marked $(,$, ) in figure $3(\mathrm{a})$ ). For $H^{\mathcal{D}}(1,3)$, the idempotents $e_{1}^{+i}$ are all well defined and $1=\sum_{i=1}^{3} e_{1}^{+i}$ is an idempotent decomposition into primitive idempotents (the corresponding simples are marked as triangles).

The set of weights for $H^{\mathcal{D}}(2,3)$ are marked with squares (the weights corresponding to the multipartitions $((2),$,$) and (,,(2))$ have been explicitly labelled, to fix the coordinate system). Note that $e_{2}^{+i}$ is divergent in case $i=2,3$. The easiest way to see what is happening at $n=2$ is to recall the $d=3$ version of the proposition in $\S$ 田:

Proposition. Fixing $k$, there are three ways to quotient to pass from $H^{\mathcal{D}}(n, 3)$ to $H^{\mathcal{D}}(n, 2)(n>0)$.

The corresponding subsets of the set of weights for $H^{\mathcal{D}}(2,3)$ lie in three straight lines - the dashed lines shown in figure 3(a). Since we have shown that the $d=2$ algebra is isomorphic to a blob algebra in each of these cases we can give a complete description. The lines marked 12 and 23 correspond to singly critical blob algebras, with reflection points the intersection points of these lines with the thick lines shown. Accordingly there are injective standard module morphisms ('reflection' morphisms) as indicated by arrows. The other blob line is a semisimple quotient (from the parameters it is nominally a singly critical case, but the reflection point lies at the outside edge of localisation of weight space to $n=2$, so there is no image of any $n=2$ weight in it). It is straightforward to show that there are no other non-trivial morphisms. Since the $d=3$ alcove structure is determined by the three $d=2$ quotients, and since the morphisms indicated are all also blob module morphisms, their location is necessarily consistent with the $d=3$ Soergel procedure.

For $H^{\mathcal{D}}(3,3)$ the three blob quotients correspond to the three dashed lines shown in figure 3(b). The reflection morphisms shown within these lines are again simply blob morphisms (NB, the reflection point on the third line now lies within the localisation of weight space). The only question, then, concerns the morphisms into the module with $A_{2}$-weight 0 . This weight is marked in the figure by the rank of the 
module, $\operatorname{Rank}\left(\left.\Delta(0)\right|_{n=3}\right)=6$ (this weight coincides with the weight for the unique simple of $H^{\mathcal{D}}(0,3)$ in our scheme, since that module is a localisation of $\left.\left.\Delta(0)\right|_{n=3}\right)$. A straightforward Frobenius reciprocity argument (using the morphisms at level $n=2$ and the generic restriction rules) shows that there are at least two homomorphisms into this module; but does not uniquely determine the domain in either case. To confirm the indicated morphisms, consider the walk basis of $\left.\Delta(0)\right|_{n=3}$ and the walk orbits of these walks - see figure 4. Note from the last of these pictures that $321 \sim^{\mathcal{G}(k)} 333$. It follows that there is a homomorphism between the corresponding standard modules by (3.12) proposition) (note that walk 321 is not sorted, but that the weaker condition of all steps in a given direction being taken consecutively is sufficient for this construction). The other claimed morphisms follow similarly.

It is worth noting that $\left.\Delta(0)\right|_{n=3}$ may generically be defined as $\left.\Delta(0)\right|_{n=3}=$ $H^{\mathcal{D}}(3,3) e_{\left(1^{3}\right)}$, where $e_{\left(1^{3}\right)}=e_{3}^{-}$is the usual $q$-antisymmetriser [55] (i.e., $\left.\Delta(0)\right|_{n=3}$ is the globalisation of the unique simple of $\left.H^{\mathcal{D}}(0,3)\right)$. This is an illuminating construct to consider in any case. The module $\mathrm{H}(3,3) e_{\left(1^{3}\right)}$ is, of course, much larger. By proposition 2.6 it has basis $\left\{X^{a} e_{\left(1^{3}\right)} \mid a \in\{0,1,2\}^{3}\right\}$. To determine a basis in our case one must use the vanishing of $e_{2}^{-i}$ (which generates a significant part of $\mathrm{H}_{\left(1^{3}\right)}$ ). This problem is dealt with elsewhere [16].

\subsection{Conjectured basis for $H^{\mathcal{D}}(n, d)$}

Consider $w \in S_{n}$, a permutation, and let $\mathbf{S}(w)$ be a corresponding reduced word in the Coxeter generators $\left\{\sigma_{i}\right\}$. The set map from $\mathbf{S}\left(S_{n}\right)$ to $\mathfrak{B}_{n}$ given by $\sigma_{i} \rightarrow g_{i}$ is an isomorphism [33]. Thus from $w \in \mathfrak{B}_{n}$ we may read off a permutation (which we will also call $w)$. Given such a permutation define symmetric relation $(w) \subset \underline{n} \times \underline{n}$ by $i(w) j$ if $i>j$ implies $w(i)<w(j)$ (and symmetry). That is, $i(w) j$ if the lines $i$ and $j$ cross in the diagram of $w$.

\section{(5.2) Claim:}

$$
\left\{X^{\left(a_{1}, a_{2}, \cdots\right)} w \in \mathfrak{C}_{n} \mid i(w) j \text { implies } a_{i} \neq a_{j}\right\}
$$

is a basis for $H^{\mathcal{D}}(n, d)$.

Idea of proof: Note that the dimensions are right (consider the RobinsonSchensted correspondence in the form in [63], or, for example, [62]). Thus it is enough to show spanning or linear independence. For the latter it is convenient to have a representation $\mathcal{R}$ of $H^{\mathcal{D}}$ (if linear independent in $\mathcal{R}$ then claim proven and $\mathcal{R}$ faithful).

The remainder of the paper is concerned with representations of $H^{\mathcal{D}}$.

\section{On representations of $b_{n}$ and $H^{\mathcal{D}}(n, d)$}

The ordinary Temperley-Lieb algebra has a powerful diagram calculus (see [49] for a review). One motivation for the introduction of the blob algebra [52] was to bring the utility of such a calculus to the representation theory of the periodic/affine algebras studied in [51] (see also [49, Ch.8], [57, 37, 27, 29] and references therein). In fact all the finite irreducible representations of these infinite dimensional algebras may be constructed using [52] (although completeness is not shown there, see [26, 54]). From the point of view of lattice Statistical Mechanics, $b_{n}$ also renders the 'seam' boundary conditions (as in [5], and cf. [46] for example) of the ice-type model [4] 

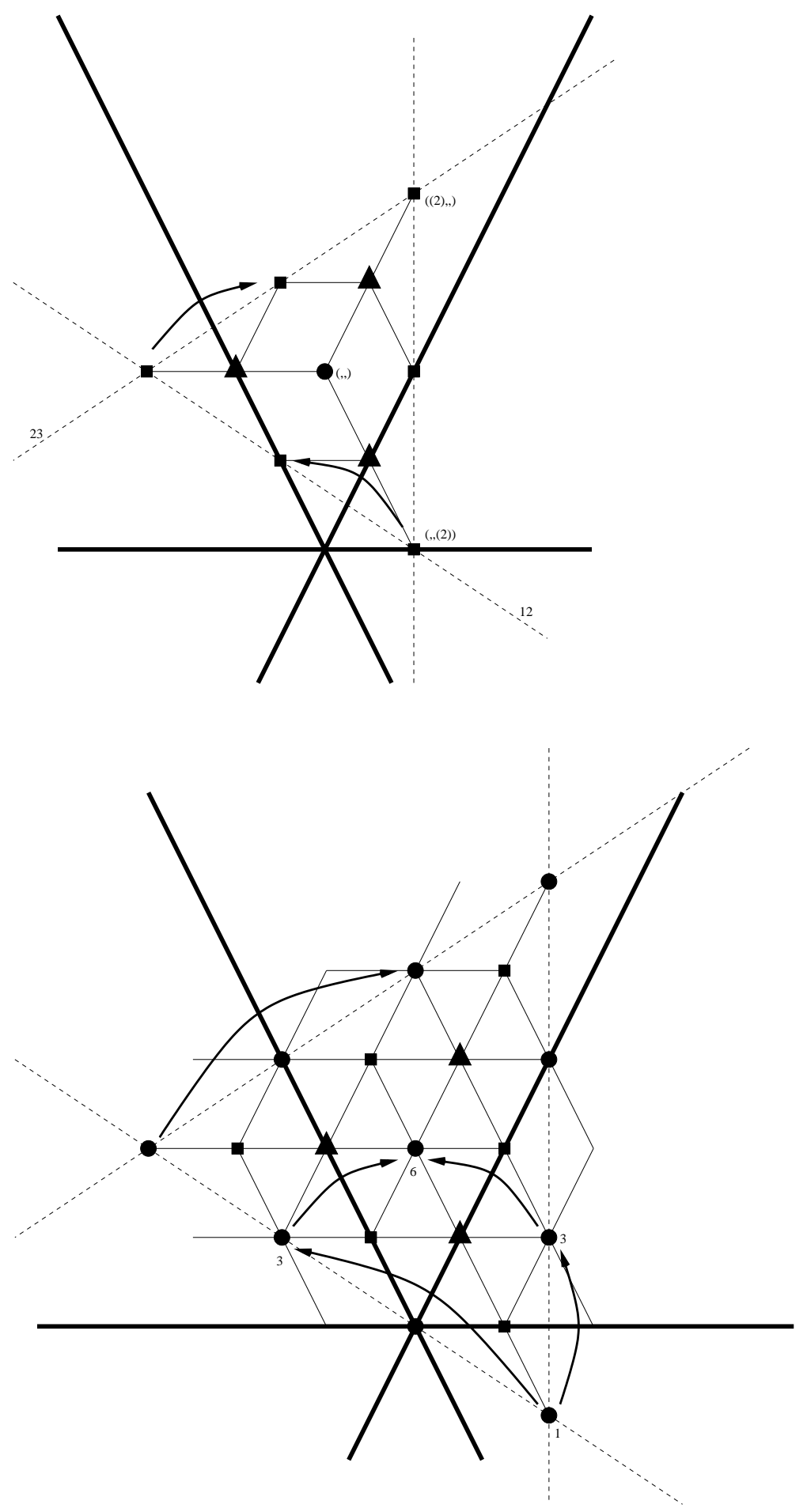

Figure 3: Weights of $H^{\mathcal{D}}(n, 3)$ in weight space formalism (a) for $n=0,1,2$; and (b) for $n=0,1,2,3$. Weight set $\Lambda(0)$ consists of the empty weight (shown as a black circle marked $(,$,$) in (\mathrm{a}) ; \Lambda(1)$ consists of the three weights marked with triangles; and so on (see main text). 

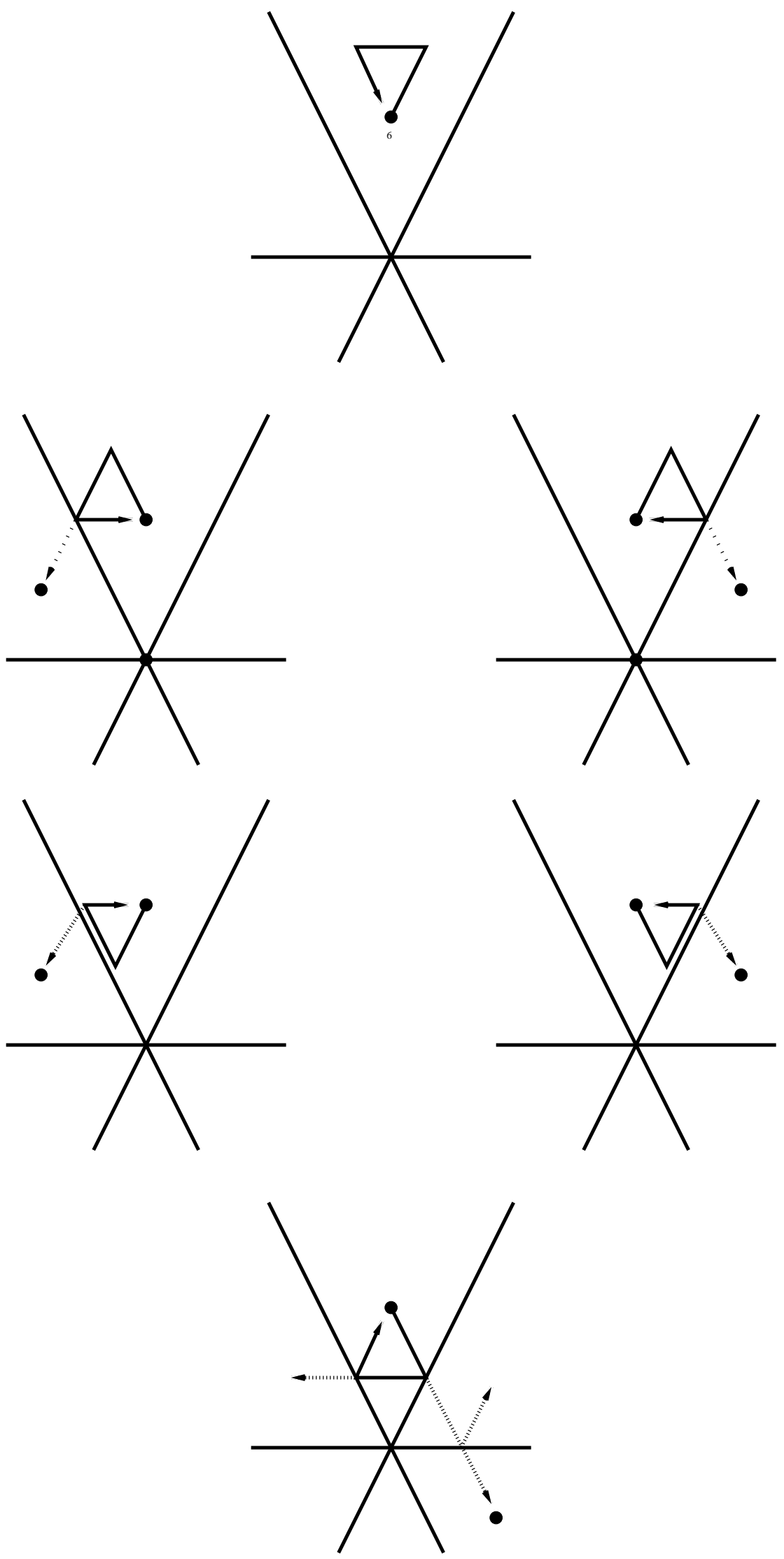

Figure 4: The six walks in $T_{0}(3)$, and their walk orbits. 
into the algebraic formalism of Temperley and Lieb [64]. Indeed there are a number of mathematical and physical reasons (in addition to the pursuit of our ingredient I2 for $\left.H^{\mathcal{D}}(-, d)\right)$ why a faithful 'tensor space' representation of the blob would be useful (cf. [52, §4], [57, 6, 21]).

By a tensor space representation of $b_{n}\left(\right.$ or $\left.H^{\mathcal{D}}(n, d)\right)$ we mean a representation for each $n$ with underlying module of form $V(n)=V_{a u x} \otimes V^{\otimes n}$ as a vector space, with $V_{\text {aux }}$ and $V$ finite dimensional vector spaces (cf. [39]); on which the generators act 'locally' (in particular $b_{n-1} \subset b_{n}$ acts trivially on the last factor $V$, so the restriction of $V(n)$ is a manifest direct sum of $\operatorname{dim} V$ copies of $V(n-1))$; and which is well defined in arbitrary specialisations.

Let $e \in H^{\mathcal{D}}(n+d, d)$ be idempotent. A module $V(n+d)$ for a tensor space representation over $K$ is globalisable by $e$ if $e$ projects the last $d$ tensor factors $V^{\otimes d} \rightarrow K$, and acts trivially on other tensor space factors.

To establish ingredient I2 generically for $H^{\mathcal{D}}(-, d)$ (i.e., that there exists idempotent $e_{d} \in H^{\mathcal{D}}(n+d, d)$ such that

$$
e_{d} H^{\mathcal{D}}(n+d, d) e_{d} \cong H^{\mathcal{D}}(n, d)
$$

cf. [12, 20]) certain generalised braid diagrams may be used [55]. However,

(6.1) Proposition. Suppose $V(n+d)$ is the module for a faithful tensor space representation of $H^{\mathcal{D}}(n+d, d)$ over $\mathbb{C}$, and is globalisable by $e_{d}$. Then equation (39) holds.

To see this note that under these assumptions the actions of $e_{d}$ and $H^{\mathcal{D}}(n, d)$ on $V(n+d)$ commute, and hence they commute in $H^{\mathcal{D}}(n+d, d)$. Thus $H^{\mathcal{D}}(n, d) e_{d} \subseteq$ $e_{d} H^{\mathcal{D}}(n+d, d) e_{d} \subseteq H^{\mathcal{D}}(n+d, d)$ is a sequence of inclusions of algebras, and $H^{\mathcal{D}}(n, d) e_{d}$ is isomorphic to $H^{\mathcal{D}}(n, d)$. Thus the action of $e_{d} H^{\mathcal{D}}(n+d, d) e_{d}$ on $V(n+d)$ would be isomorphic to the $H^{\mathcal{D}}(n, d)$ action on $V(n)$. Since the latter has a trivial kernel, this would establish equation (39) in general.

Any faithful tensor space representation in which $X$ acts non-trivially only in the first normal tensor factor $V$ (and an otherwise redundant factor $V^{\text {aux }}$ ) would be a likely candidate, because of the way ingredient I2 works in the $A_{n}$ case [36]. Let us briefly review this.

(6.2) The ordinary $H_{n}$ action (dual to that of $U_{q} s l_{N}$ ) on $V_{N}^{n}$ is

$$
\begin{gathered}
M_{N}^{q}: A_{n} \text {-braid } \rightarrow \operatorname{End}\left(V_{N}^{n}\right) \\
g_{i} \mapsto 1 \otimes 1 \otimes \ldots \otimes \mathcal{M}^{q} \otimes 1 \otimes 1 \otimes \ldots \otimes 1
\end{gathered}
$$

where 1 denotes the unit matrix; and $\mathcal{M}^{q}$ (the $i^{\text {th }}$ factor) is given by

$$
\left.\mathcal{M}^{q}\right|_{N=2}=\left(\begin{array}{cccc}
q & 0 & 0 & 0 \\
0 & q-q^{-1} & -1 & 0 \\
0 & -1 & 0 & 0 \\
0 & 0 & 0 & q
\end{array}\right)
$$




$$
\left.\mathcal{M}^{q}\right|_{N=3}=\left(\begin{array}{cccccccccc}
q & 0 & 0 & 0 & & & & & \\
0 & -q^{-1}+q & 0 & -1 & 0 & & & & \\
0 & 0 & -q^{-1}+q & 0 & 0 & 0 & -1 & 0 & \\
0 & -1 & 0 & 0 & 0 & 0 & 0 & 0 & 0 \\
0 & 0 & 0 & 0 & q & 0 & 0 & 0 & 0 \\
0 & 0 & 0 & 0 & 0 & -q^{-1}+q & 0 & -1 & 0 \\
0 & 0 & -1 & 0 & 0 & 0 & 0 & 0 & 0 \\
0 & 0 & 0 & 0 & 0 & -1 & 0 & 0 & 0 \\
0 & 0 & 0 & 0 & 0 & 0 & 0 & 0 & q
\end{array}\right)
$$

and so on. Each such obeys a quadratic local relation with coefficients in $R=$ $\mathbb{Z}\left[q, q^{-1}\right]$ (specifically $\left.M_{N}\left(\left(g_{1}-q\right)\left(g_{1}-q^{-1}\right)\right)=0\right)$.

Putting $U_{i}=g_{i}-q$ we have

$$
M_{2}^{q}\left(U_{i} U_{i \pm 1} U_{i}-U_{i}\right)=0
$$

so $M_{2}^{q}$ factors through $T_{n}(q)$ (in fact it is faithful on $T_{n}(q)$, i.e. $T_{n}(q)=H_{n}^{2}(q)$ ).

Recall from $\$ 1.2$ that $e_{N}^{-}$denotes the $H_{N} q$-symmetriser (normalisable as an idempotent provided that [N]! is invertible in $K[49])$. For $n \geq N, V_{N}^{n}$ is the tensor space module for $H_{n}^{N}(q)$. It is easy to check that $V_{N}^{n}$ is globalisable by $e_{N}^{-}$.

(6.3) Recall that the $T_{n}$ action on $V_{2}^{n}$ breaks up directly, over any field, into summands $\mathcal{P}_{\lambda}$ of fixed 'charge' or weight $\lambda \in \mathbb{N}_{0}$, and then

$$
\mathcal{P}_{\lambda}=+_{\mu \geq \lambda} \Delta_{\mu}
$$

(generically a direct sum).

It is desirable not only to have representations of $b_{n}$ that act on tensor space, but also that they preserve some version of this charge conservation - i.e. they are a direct sum of analogues of permutation representations. (Note for example that the tensor space representation in [52, §4] is neither full tilting [21] nor charge conserving.)

One way to proceed is to search for maps from $b_{n}$ to $T_{n^{\prime}}\left(\right.$ some $\left.n^{\prime}\right)\left(\right.$ resp. $H^{\mathcal{D}}(n, d)$ to $H_{n^{\prime}}^{d}$ ), and hence obtain $b_{n}$-modules by restriction of $M_{2}^{q}$. Another possibility is to enrich suitable $T_{n^{\prime}}$-modules with the property of $b_{n}$-module by determining an action of the blob operator on them. We begin by investigating the latter.

\subsection{Generalised bialgebra construction}

Let $(M, \circ, e)$ be a finite monoid, and $A$ a $K$-algebra with basis $M$ and multiplication defined on this basis by $m_{1} m_{2}=k_{m_{1}, m_{2}}(\mathbf{q}) m_{1} \circ m_{2}$ where $\mathbf{q}$ is some set of parameters and $k_{12}(\mathbf{q}) \in K$. (The possibilities for the coefficients will in general by constrained by $M$,

$$
\begin{gathered}
m_{1}\left(m_{2} m_{3}\right)=m_{1} k_{m_{2}, m_{3}}(\mathbf{q})\left(m_{2} \circ m_{3}\right)=k_{m_{2}, m_{3}}(\mathbf{q}) k_{m_{1}, m_{2} \circ m_{3}}(\mathbf{q}) m_{1} \circ\left(m_{2} \circ m_{3}\right) \\
=k_{m_{1}, m_{2}}(\mathbf{q})\left(m_{1} \circ m_{2}\right) m_{3}=k_{m_{1}, m_{2}}(\mathbf{q}) k_{m_{1} \circ m_{2}, m_{3}}(\mathbf{q})\left(m_{1} \circ m_{2}\right) \circ m_{3}
\end{gathered}
$$

so $k_{m_{2}, m_{3}}(\mathbf{q}) k_{m_{1}, m_{2} \circ m_{3}}(\mathbf{q})=k_{m_{1}, m_{2}}(\mathbf{q}) k_{m_{1} \circ m_{2}, m_{3}}(\mathbf{q})$, but there are plentiful solutions - for example, any finite group algebra.) Suppose there is a triple of points in parameter space for which

$$
k_{m_{1}, m_{2}}\left(\mathbf{q}^{\prime}\right) k_{m_{1}, m_{2}}\left(\mathbf{q}^{\prime \prime}\right)=k_{m_{1}, m_{2}}(\mathbf{q})
$$




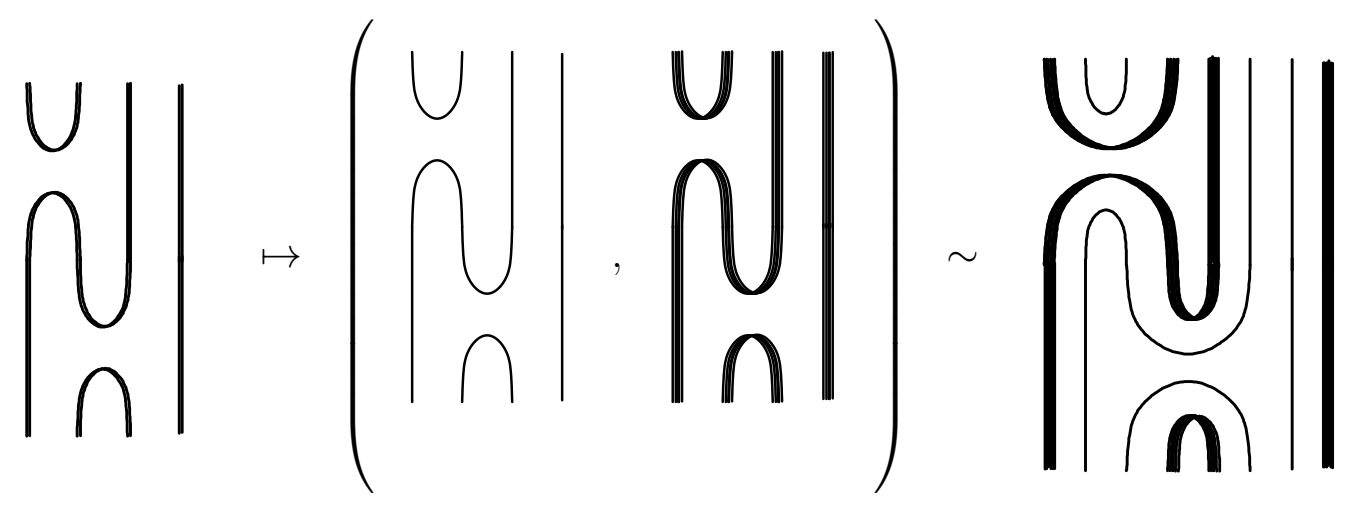

Figure 5:

for all $m_{1}, m_{2}$, then $A$ has a kind of generalised coproduct:

$$
\begin{aligned}
& A \hookrightarrow A^{\prime} \times A^{\prime \prime} \\
& m \mapsto(m, m)
\end{aligned}
$$

making it a kind of generalised bialgebra, since

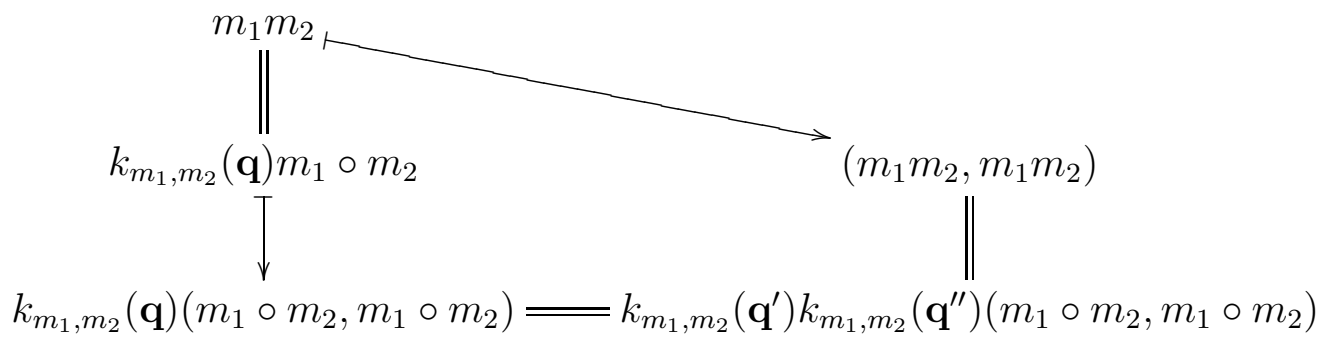

(cf. [38, §1.1.3(iv)], for example). In particular the coproduct is an algebra morphism and if a submanifold $S$ of parameter space can be found for which each pair $\mathbf{q}^{\prime}, \mathbf{q}^{\prime \prime} \in S$ has a $\mathbf{q} \in S$ satisfying equation(42) then the sum over all $\mathbf{q} \in S$ of categories of (left) modules is closed under tensor product. (The example of group algebras is the usual bialgebra and tensor product.)

It is easy to show, using the diagram calculus (or via a mild generalisation of the T-L diagram variant of cabling [47, $\S \mathrm{A}(\mathrm{iii})]$ ), that $T_{n}(q)$ is an algebra of this type, with $\mathbf{q}=\{q\}$. The diagram in figure 5 illustrates the coproduct on $U_{1} U_{2} \in T_{4}(q)$, using lines of different thickness for different $q$. The cabling-like visualisation of the two factors, in which they are embedded in a single pseudodiagram, is possible because the thin and thick lines are arranged into subdiagrams which never meet in any composition. It will be evident that the set of conditions (42) include $-[2]_{q}=$ $[2]_{q^{\prime}}[2]_{q^{\prime \prime}}$ in this case (consider a composition in which a closed loop arises, such as $\left.U_{1} U_{1}\right)$; and that this is the only non-trivial condition. Through the cabling picture we may pass to another visualisation, in which the thinner lines have been reflected in a vertical line at the left edge of the diagram (cf. [65]), as illustrated in figure 6. There is no significant difference between these two visualisations, except that it is perhaps slightly easier to describe the construction of certain tensor product representations explicitly using the reflected form, as we will see. Again in the reflected form we may view the picture as a single pseudodiagram (but again there is no sense in which the right and left hand sides ever touch). 


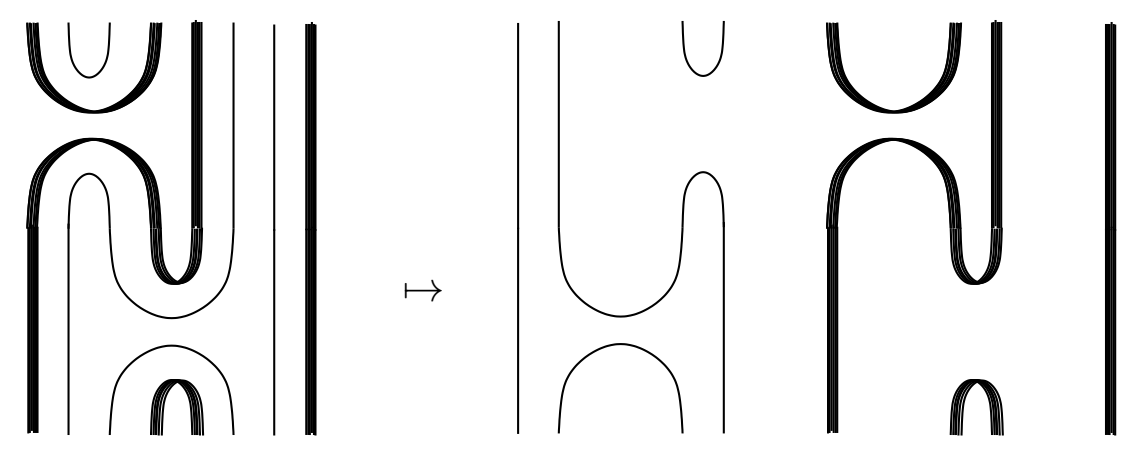

Figure 6:

For example we may tensor together two tensor space representations in the form:

$$
\begin{gathered}
\rho: T_{n}(q) \longrightarrow \operatorname{End}\left(V_{2}^{2 n}\right) \\
\rho: U_{i} \mapsto M_{2}^{s}\left(U_{n-i}\right) M_{2}^{t}\left(U_{n+i}\right)
\end{gathered}
$$

Here the set of conditions (42) reduce to $-[2]_{q}=[2]_{s}[2]_{t}$ via, e.g.,

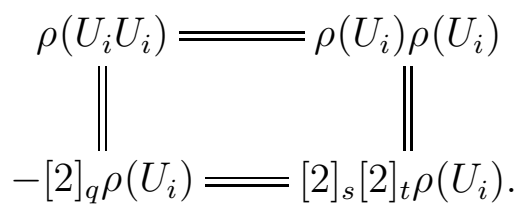

Set

$$
\mathcal{U}^{q}(x)=\left(\begin{array}{cccc}
0 & 0 & 0 & 0 \\
0 & q & 1 & 0 \\
0 & 1 & q^{-1} & 0 \\
0 & 0 & 0 & x
\end{array}\right)
$$

and $\mathcal{U}^{q}=\mathcal{U}^{q}(0)\left(\mathrm{cf}\right.$. 18). Just as $M_{2}^{r}\left(U_{i}\right)$ is a matrix acting trivially on every tensor factor except the $i^{t h}$ and $(i+1)^{t h}$, where it acts as $-\mathcal{U}^{r}$, so let $M_{2}^{r, x}\left(U_{i}\right)$ denote a matrix differing from this only in acting like $-\mathcal{U}^{r}(x)$ in that position.

Note that

$$
\left(\mathcal{U}^{s} \otimes \mathcal{U}^{t}\right)\left(1 \otimes \mathcal{U}^{r}(x) \otimes 1\right)\left(\mathcal{U}^{s} \otimes \mathcal{U}^{t}\right)=\left(\frac{r}{s t}+\frac{s t}{r}+x \frac{t}{s}\right)\left(\mathcal{U}^{s} \otimes \mathcal{U}^{t}\right)
$$

for any $r, s, t, x$ (an explicit calculation).

(6.4) Proposition. Fix $q, m$, put $q=e^{i \mu_{q}}, u_{0}=[m]_{q} e_{-}$, and choose $r, s, t$ such that

$$
\begin{gathered}
-\cos \left(\mu_{q}\right)=2 \cos \left(\mu_{s}\right) \cos \left(\mu_{t}\right) \\
-\frac{\sin \left((m-1) \mu_{q}\right)}{\sin \left(m \mu_{q}\right)}=\frac{\cos \left(\mu_{s}+\mu_{t}-\mu_{r}\right)}{\cos \left(\mu_{r}\right)} .
\end{gathered}
$$

(NB, exclude $m=0$ and caveat $q=1$. A convenient realisation is $r=i(-q)^{m}$, $s=-i \sqrt{i q}, t=-\sqrt{i q}$, i.e. $\mu_{r}=m\left(\mu_{q}+\pi\right)+\frac{\pi}{2}, \mu_{s}=\frac{\mu_{q}+\pi}{2}-\frac{3 \pi}{4}, \mu_{t}=\frac{\mu_{q}+\pi}{2}+\frac{3 \pi}{4}-s o$ rational $\frac{\mu_{q}}{\pi}$ and $m$ gives rational $\frac{\mu_{r}}{\pi}, \frac{\mu_{s}}{\pi}, \frac{\mu_{t}}{\pi}$.) Then there is an algebra homomorphism

$$
\rho_{0}: b_{n}(q, m) \longrightarrow \operatorname{End}\left(V_{2}^{2 n}\right)
$$




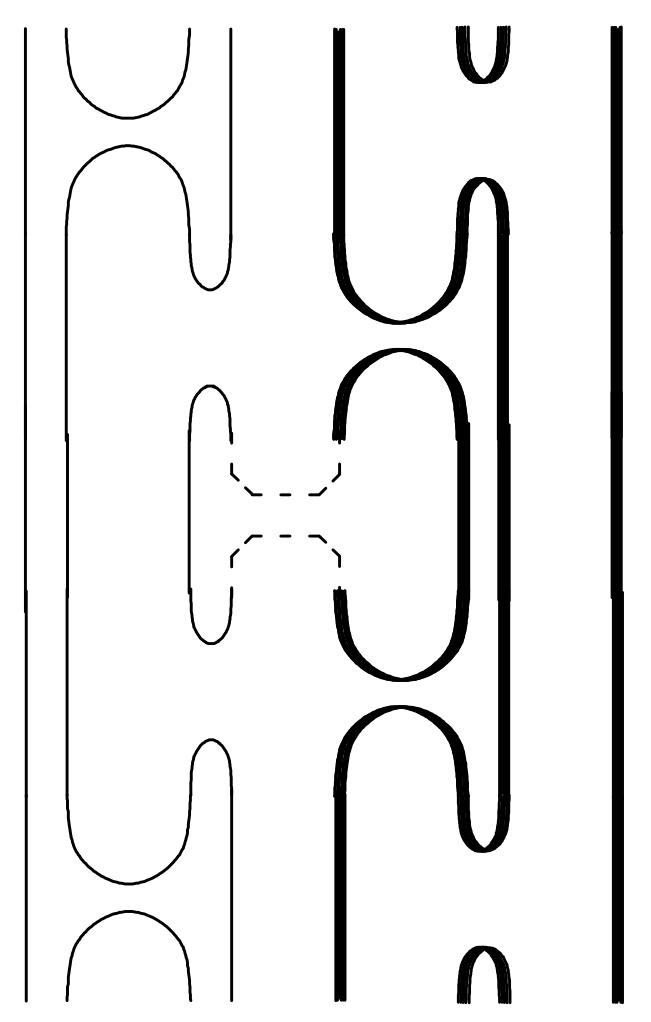

Figure 7:

given by

$$
\begin{gathered}
\rho_{0}: e_{-} \mapsto \frac{1}{[2]_{r}} M_{2}^{r}\left(U_{n}\right) \\
\rho_{0}: U_{i} \mapsto M_{2}^{s}\left(U_{n-i}\right) M_{2}^{t}\left(U_{n+i}\right) .
\end{gathered}
$$

Proof: We may readily verify $\rho_{0}\left(e_{-} e_{-}=e_{-}\right)$. The relations for $T_{n}(q) \subset b_{n}$ may be checked in $\rho$, i.e. as above $\left(\mathrm{NB}[m]_{q}=\frac{\sin \left(m \mu_{q}\right)}{\sin \left(\mu_{q}\right)}\right)$. There remains $U_{1} e_{-} U_{1} \propto U_{1}$ (relation(31)). This is validated by the explicit calculation in equation(43):

$$
U_{1} e_{-} U_{1}=\frac{[m-1]_{q}}{[m]_{q}} U_{1} \mapsto-\frac{\left(\frac{r}{s t}+\frac{s t}{r}\right)}{[2]_{r}} \rho_{0}\left(U_{1}\right) .
$$

Another way to see this is to note that, in tensor space, equation (43) allows us to make sense of an extension of the pseudodiagrams in figure 6 in which the left and right hand sides meet as in figure 7 (specifically, this figure may be replaced by a scalar multiple of one in which the loop composed of mixed line segments is omitted).

(6.5) There is a similar homomorphism $\rho_{s}$ which simply replaces equation(46) with

$$
\rho_{s}: e_{-} \mapsto \frac{1}{[2]_{r}} M_{2}^{r,[2]_{r}}\left(U_{n}\right)
$$

and equation(45) with

$$
-\frac{\sin \left((m-1) \mu_{q}\right)}{\sin \left(m \mu_{q}\right)}=\frac{\cos \left(\mu_{s}+\mu_{t}-\mu_{r}\right)}{\cos \left(\mu_{r}\right)}+e^{i\left(\mu_{t}-\mu_{s}\right)}
$$



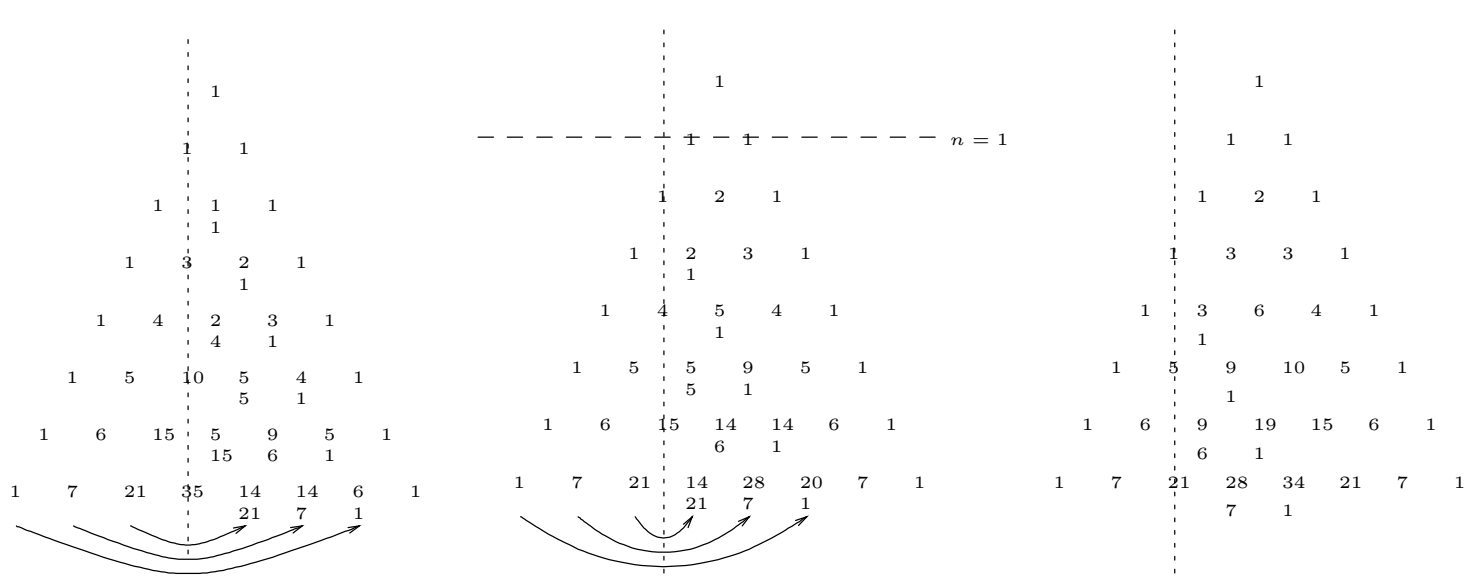

Figure 8: The Pascal triangle of $b_{n}$ standard modules (layers $n=0,1, \ldots, 7$ are shown) complicated at $m \in \mathbb{Z}$ by reflection homomorphisms. Here we show, by their dimensions, the composition factors of each standard in cases $m=-1,-2,-3$.

This mild complication has the merit that the blob/box symmetry of the algebra [52] maps this representation to one of the same type.

The isomorphism of $b_{n}$ to its opposite (note the symmetry of the relations under writing back to front) provides an automorphism which is fixed by $\rho_{0}$ (the representations of the generators are symmetric matrices), thus $\rho_{0}$ is contravariant self-dual. The same is true of $\rho_{s}$.

These very exciting representations merit further study. Cox, Martin and RyomHansen have recently shown [16] that they are generically faithful. Other intriguing questions are: Are they (full) tilting? How do they generalize to higher $d$ ? The latter question is not trivial. We have made considerable use of the $\mathrm{T}-\mathrm{L}$ diagram calculus here, and there is no such powerful tool in evidence for higher $d$, short of the braid group itself. The remainder of the paper is essentially concerned with addressing this question.

\subsection{Other constructions}

The blob algebra $b_{n}(q, m)$ is (at least) singly critical when $m \in \mathbb{Z}^{+}$, cf. section 1.1 - if $q$ is not a root of unity then the procedure there still works, but with $l$ set unboundedly large. This means that the usual Pascal triangle of standard modules 52 is complicated by at least one wall of 'reflection' homomorphisms from outside to inside across the alcove wall at $m$, as exemplified in figure 8. There is a suggestive combinatorial coincidence with $T_{n}$ manifest in the dimension of heads of the blob standard modules in certain singly critical (i.e. $m \in \mathbb{Z}$ ) cases. This starts with the $m= \pm 1$ cases, where there is a $b_{n}$ quotient given by $e_{-} \mapsto 0$ (resp. 1). It follows immediately from the relations that this quotient is isomorphic to $T_{n}$ (and so the coincidence is explained). Figure 8 exhibits a similar phenomenon at $m=-2$. These may be regarded as special cases of a more general 'braid construction'.

In the next sections we describe this braid construction, and review aspects of the connection between the blob algebra and periodic (and affine) systems which lead to other useful maps from $b_{n}$ (and other $\mathrm{H}(n, d)$ quotients) into ordinary Hecke quotients, and hence into tensor space. 


\section{Braids and the blob approach to periodic sys- tems}

In this paper the 'A-type braid group' $A$-braid is the group of braidings of a row of initially vertical strings numbered from the top left: $1,2, \ldots$, which braidings are trivial on all but finitely many strings. The subgroup $A_{n}$-braid acts trivially on all but the first $n$ strings (so $A_{0}$-braid $=A_{1}$-braid $\subset A_{2}$-braid $\subset \ldots$ ). Thus $H_{n}$ is a quotient of $\mathbb{C} A_{n}$-braid, and $g_{i}$ is the braid in which string $i$ crosses over string $i+1$. Evidently such elements, and inverses, generate the group [45, 8]. Let (-) : $A_{n}$-braid $\rightarrow A_{n}$-braid be the automorphism given by $g_{i} \mapsto g_{n-i}$ [49, §5.7.2] (cf. [65]).

Occasionally we shall need to refer to the group of braidings of precisely $n$ strings (as for example in the Young subgroup construction — see [49, §13.1 p323]). This group is obviously isomorphic to $A_{n}$-braid, and we will distinguish them only by context. Let $1^{m}$ denote the identity element on precisely $m$ strings, and

$$
1_{C}^{m}: A_{n} \text {-braid } \rightarrow A_{m n} \text {-braid }
$$

the corresponding cabling morphism (replace each string with $m$ parallel strings).

Let $\Delta: A_{n}$-braid $\rightarrow A_{n}$-braid $\times A_{n}$-braid be the group comultiplication. Let $Y: A_{n}$-braid $\times A_{n}$-braid $\hookrightarrow A_{2 n}$-braid be the natural 'Young' embedding, extended to (a version of) the full braid group by extending the numbering of strings to $\mathbb{Z} \backslash\{0\}$ - i.e. essentially the full line not the half line - and placing the second copy of $A_{n}$-braid on the 'minus' side. Let $F$ be the map back to the full braid group proper got by folding the left hand side of the plane over onto the right hand side at a point slightly shifted from the origin (so that each negatively numbered string starting point lies just to the left of its positively numbered version and the system is bounded on the left again), then renumbering - see figure 9 . Let $S$ be the map back to $A$-braid got by renumbering $i \mapsto i+n+1(i<0)$ and $i \mapsto i+n$ $(i>0)$ and discarding all strings numbered less than 1 . Now define a map $1_{F}^{2}$ from $A_{n}$-braid $\rightarrow A_{2 n}$-braid by commutativity of:

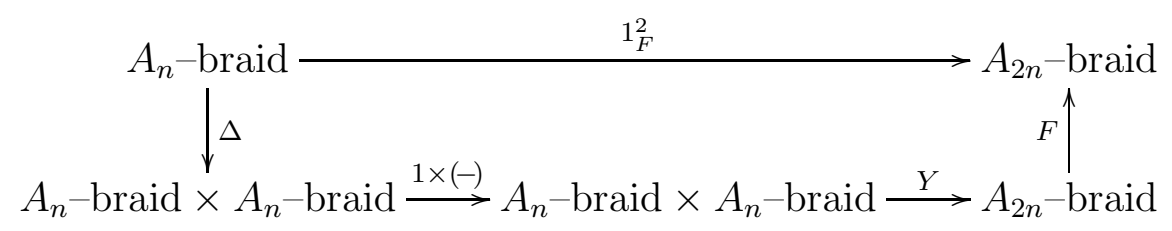

and similarly for $1_{S}^{2}$. The map $1_{F}^{2}$ is similar also to the $m=2$ cabling morphism in that each string now has a partner running parallel to it, but the over/under information is not the same.

(7.1) We now recall certain constructions from [49, §5.7] (some changes of notation will be necessary). Let $\mathbb{C} \hat{A}_{n}$-braid denote the algebra associated to affine graph $\hat{A}_{n}$ there (strictly speaking only every other such graph has a pregraph, but this need not concern us); let $\hat{A}_{n}$-braid denote the underlying group (i.e. the affinization of the ordinary braid group); and let $g$. denote the 'extra' generator associated to the affinizing vertex in $\hat{A}_{n}$ cf. $A_{n-1}$. Define

$$
G=g_{1} g_{2} \ldots g_{n-1}
$$

in $A_{n}$-braid (the element in which string 1 crosses over strings 2 to $n$ ); and note that $G g_{i} G^{-1}=g_{i+1}(i<n-1)$. 

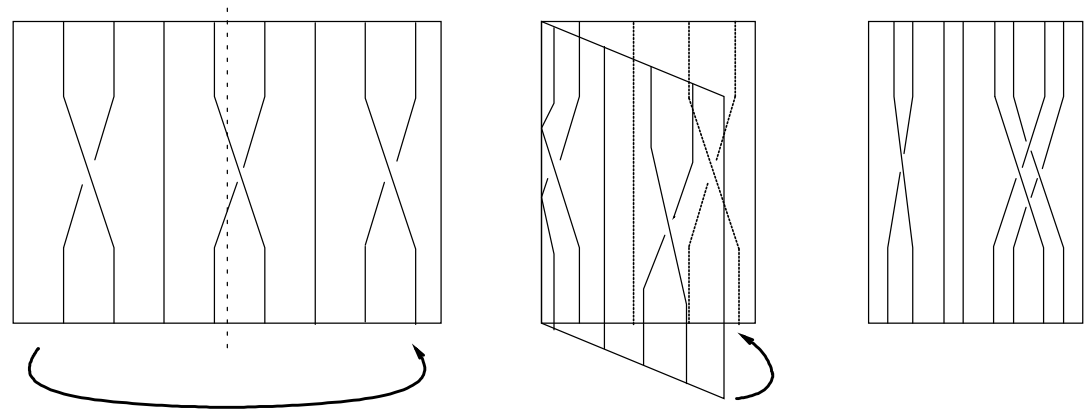

Figure 9: Illustration of the folding injection of $A_{n}$-braid into itself.

Proposition. 49 There is a homomorphism $\phi_{0}: \hat{A}_{n}$-braid $\mapsto A_{n}$-braid given by identification on the $A_{n}$-braid subalgebra and $g . \mapsto G g_{n-1} G^{-1}$.

As noted for example in [47, §3] there are actually a number of closely related ways of building representations of the affine (or periodic) case, corresponding to the choice of periodic boundary conditions (the 'cohomological seam') in a physical system. This was systematized, in [52, §3], by the introduction of the idempotent blob generator $e_{-}$. Using this one builds an invertible generator $g_{-}=1+a e_{-}(a$ a suitable constant) obeying

$$
g_{-} g_{1} g_{-} g_{1}=g_{1} g_{-} g_{1} g_{-}
$$

(see also proposition (4.4)) and defines $G_{-}=g_{-} G$. Then

$$
\text { g. } \mapsto G_{-} g_{n-1}\left(G_{-}\right)^{-1}
$$

defines a generalisation $\phi_{a}$ of $\phi_{0}$ for each suitable choice of $a, y_{e}$ (see [52, eq.(25)]).

Note that neither equation(田) nor the blob construction for $g_{-}$are needed to verify the map in eqn.(50); only equation(49) is necessary. Thus the map generalises to $H(n)$, and even to the level of braids:

(7.2) The connection between the $B$-type and periodic systems now follows, in as much as $B_{n}$-braid may be realized as the group of braids on the cylinder, whereupon $\pi=g_{n-1} g_{n-2} \ldots g_{1} g_{0}$ is the braid got from the identity braid by turning the bottom edge of the cylinder through one vertex clockwise (i.e. so as to take vertex 1 into vertex 2 , and so on); and $\pi^{\prime}$, the corresponding generalisation of $G_{-}$(i.e. with $g_{-}$ replaced by $g_{0}$ ), is the anticlockwise turn. Thus $B_{n}$-braid may be thought of as having affine $\hat{A}_{n}$-braid as a subgroup — with $g .=\pi g_{1} \pi^{-1}=\pi^{\prime} g_{n-1}\left(\pi^{\prime}\right)^{-1}$, cf. [52, $\S 3]$.

\subsection{Homomorphisms of $B_{n}$-braid to $A_{n^{\prime}}$-braid}

(7.3) Let $A=A_{n}$-braid and $\underline{n}=\{1,2, \ldots, n\}$. For $b \in A$ and $i \in \underline{n}$ define $b(i)$ to be the final position of string $i$ in $b$.

Let $p$ be any partition of (equivalently, equivalence relation on) $\underline{n}$. Then for each such $p$ there is a subset of $A=A_{n}$-braid such that $b \in A$ implies $b(i) \sim i$. This subset is a subgroup - call it $p-A_{n}$-braid, or just $p$-braid. E.g. if $p$ is the 'trivial' relation $(\{\underline{n}\})$ then $p$-braid $=A$; if $p$ is the identity relation then $p$-braid is the pure braid group (the normal subgroup whose quotient is the permutation action on $\underline{n}$, $b(i)$, described above). 


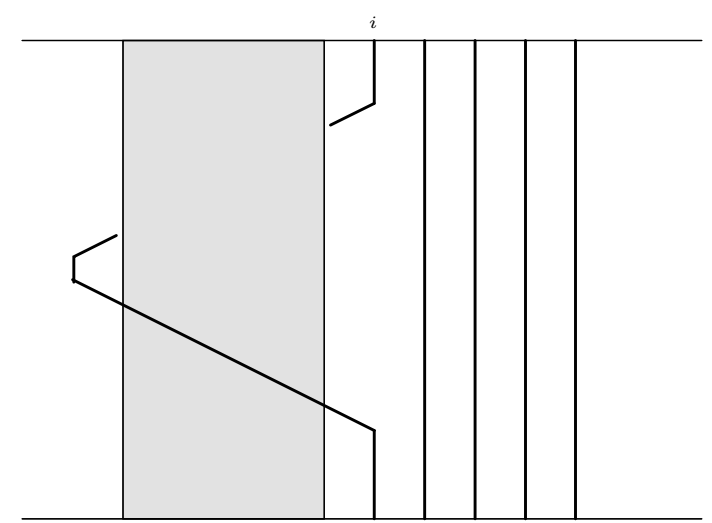

Figure 10: $L_{i}$ - here the shaded area represents the identity braid on the first $i-1$ strings.

For convenience when dealing with general $n$ we will describe a partition for which each $i>m$, some $m$, is in the same part by only giving the other parts. Thus \{\} -braid $=\{\underline{n}\}$-braid $=A$; and $\{\{1\}\}$-braid $=: A^{\prime}$ is the group which is pure on string 1 .

(7.4) Let $L_{i} \in A_{n}$-braid be the pure braid which takes string $i$ behind all earlier strings then back in front of them (i.e. $L_{1}=1$ and $L_{i+1}=g_{i} L_{i} g_{i}$ ). See figure 10 .

Note that in the Hecke algebra quotient $L_{i}$ is the image of $\left.X_{i} \in \mathrm{H}(n, 1)\right|_{\lambda_{1}=1}$; that $L_{i} L_{j}=L_{j} L_{i}$ and that $C_{n}=\prod_{i=1}^{n} L_{i}$ is the ('clockwise' or $g_{i}$-built) pure twist element of $A_{n}$-braid, denoted $M^{2}$ in [49, §5.7.2].

(7.5) Proposition. Let $b$ be any element of $A_{m-1}$-braid. Then there is an injective group homomorphism

$$
\begin{aligned}
f_{b}^{m}: B_{n} \text {-braid } \longrightarrow & \{\underline{m-1}\}-A_{n+m-1} \text {-braid } \\
g_{0} & \mapsto C_{m} b \\
g_{i} & \mapsto g_{i+m-1} .
\end{aligned}
$$

Proof: consider figure 11. This checks the key relation explicitly.

For example, $f_{1}^{2}$ is $g_{0} \mapsto g_{1} g_{1}, g_{1} \mapsto g_{2}$ and so on. $f_{1}^{1}$ is $g_{0} \mapsto 1, g_{i} \mapsto g_{i}(i>0)$.

(7.6) There is a group homomorphism extending the $1_{C}^{2}$ cabling morphism (cf. [49, Ch.13]; note also [17):

$$
\begin{aligned}
1_{C}^{2}: B_{n} \text {-braid } & \longrightarrow A_{2 n} \text {-braid } \\
g_{0} & \mapsto g_{1} \\
g_{i} & \mapsto 1_{C}^{2}\left(g_{i}\right) .
\end{aligned}
$$

There is a similar homomorphism extending the $1_{S}^{2}$ morphism:

$$
\begin{aligned}
1_{S}^{2}: B_{n} \text {-braid } & \longrightarrow A_{2 n} \text {-braid } \\
g_{0} & \mapsto g_{n} \\
g_{i} & \mapsto g_{n-i} g_{n+i}
\end{aligned}
$$

(cf. figure 9); and another extending $1_{F}^{2}$. 


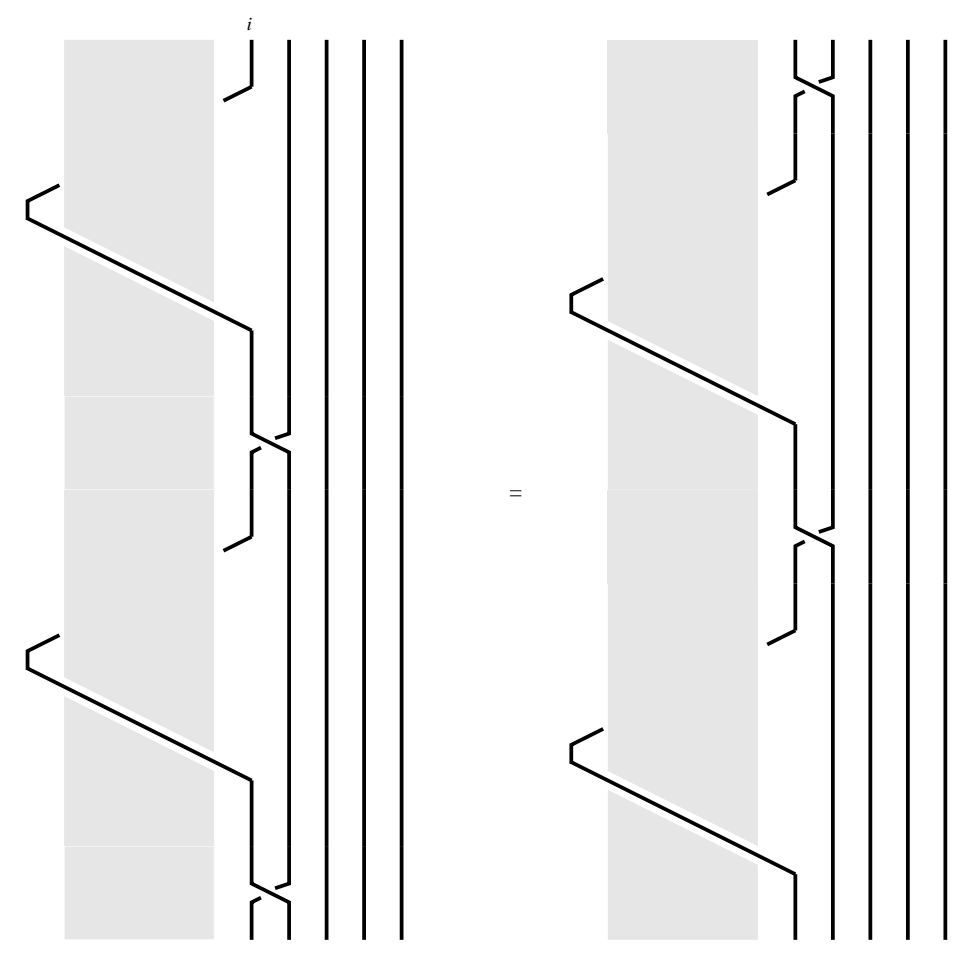

Figure 11: Schematic for generalisations of $L_{i} g_{i} L_{i} g_{i}=L_{i} L_{i+1}=L_{i+1} L_{i}=g_{i} L_{i} g_{i} L_{i}$ as in $f_{b}$ ( $b$ lives in the shaded region).

\section{On tensor space representations of $\mathbf{H}(n, d)$}

The constructions above allow us to build representations of $B_{n}$-braid from type$A$ representations (and if these are charge conserving tensor space representations then these properties will be preserved, in some sense, as will globalisability). Our strategy now in searching for maps from $b_{n}$ to $T_{n}$ (and generalisations to $d>2$ ) may be summarized by the following picture.

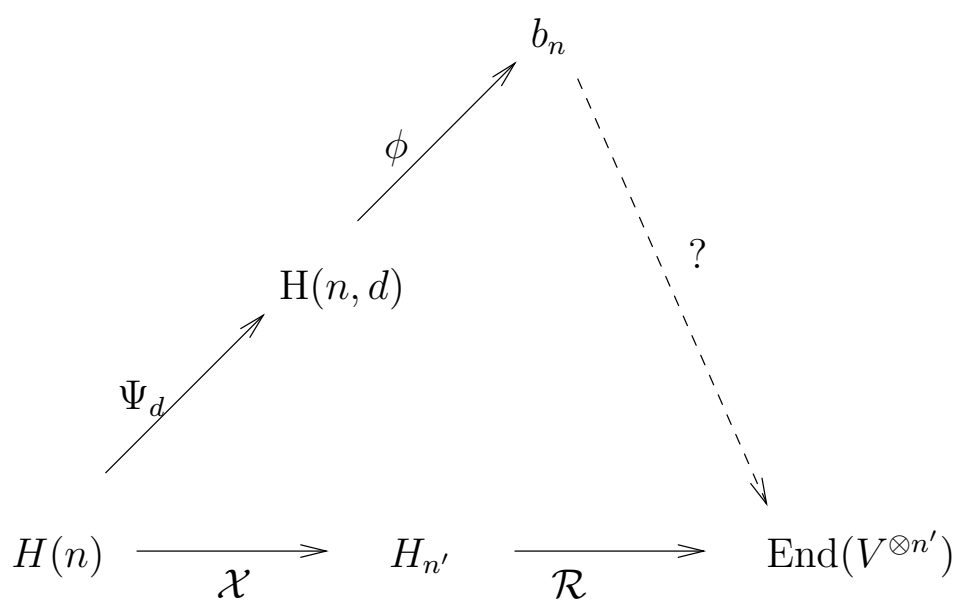

The Northeast pointing maps are the canonical quotients from sections 1 and 1 (we will call the combined map $\eta$ ); the map $\mathcal{R}$ is the ordinary representation on tensor space; and the dotted line is the desired map occurring if $\mathcal{R} \circ \mathcal{X}$ factors through $b_{n}$. The exercise is to find among the maps $\mathcal{X}$ those for which $\mathcal{R} \circ \mathcal{X}$ so factors. (As 
we will see, the candidates we noted in $\S 6.2$ for maps from $b_{n}$ to $T_{n}$ are just special cases of the simple representations $f_{b}^{m}$ of $B_{n}$-braid above.)

By (6.2) each $B$-braid quotient of form

$$
B_{n} \text {-braid } \stackrel{f_{b}^{m}}{\longrightarrow} A_{m+n} \text {-braid } \stackrel{M_{N}}{\longrightarrow} V_{N}^{m+n}
$$

('auxiliary space' construction) or

$$
B_{n} \text {-braid } \stackrel{1^{2}}{\longrightarrow} A_{2 n} \text {-braid } \stackrel{M_{N}}{\longrightarrow} V_{N}^{2 n}
$$

('cabling related' construction) factors through a partial specialisation of $\mathrm{H}(n, d)$ (some $d$ ) in which the $g_{0}$ (i.e. $X$ ) eigenvalues are determined in terms of $q$, but $q$ is indeterminate. Obviously the $M_{2} \circ f_{1}^{2}$ quotient obeys a quadratic $f\left(g_{0}\right)=0$, so $d=2$, so it factors through some specialisation of $\operatorname{Hecke}_{B}(n)$; but $f\left(g_{0}\right)$ has coefficients in $\mathbb{Z}\left[q, q^{-1}\right]$, so still this is not generic (Hecke ${ }_{B}$ has two parameters, $q$ and $Q$, say).

Since all our maps $M_{2} \circ \mathcal{X}$ map into the $T_{n}$ action on $V_{2}^{n}$ (for some $n$ ), their image breaks up at least as far as in eqn.(41).

Possible next steps here are: (i) to investigate the generic irreducible content of the $f_{b}$ representations (of whatever specialisation of $\mathrm{H}(n, d)$ they might provide); and (ii) to investigate what portion of parameter space is actually accessible by this construction (i.e. what eigenvalues of $g_{0}$ we can realise by varying $b$ ). Fixing $q$, this portion is discrete, i.e. of measure zero, but then so is the (at least singly critical) portion we are most interested in, so it is not necessarily too restricted.

$\mathrm{NB}$, if we want to access a dense subset of parameter space this cannot be via $M_{N} \circ f_{b}^{m}$, since this depends continuously only on $q$. Instead we could look at maps ending on, say, $\left.\otimes_{i=1}^{d} V_{N}^{n}\right|_{q=q_{i}}$.

\subsection{On cabling related maps}

Underlying the map $1_{F}^{2}$ is the full range of direct product representations of $A_{n}$-braid. In particular we can regard $\otimes_{i} M_{N}^{q_{i}}$ as a representation of $A_{n}$-braid. In general we may not assume that these representations will factor through any particular Hecke quotient, but if one does then it could provide a generalisation of the extension of $1_{F}^{2}$ to $B_{n}$-braid. We form $R^{\otimes}\left(g_{i}\right)=M_{N}^{q}\left(g_{n-i}\right) \otimes M_{N}^{r}\left(g_{n+i}\right)$ and try, say, $R^{\otimes}\left(g_{0}\right)=1 \otimes M_{N}^{s}\left(g_{n-i}\right) \otimes 1$ and compute $R^{\otimes}\left(g_{0} g_{1} g_{0} g_{1}-g_{1} g_{0} g_{1} g_{0}\right)$. In this particular case, with $N=3$, the image vanishes only when $q=r=s$ (a brute force calculation). An analogous deformation of the extended $1_{C}^{2}$ map at $N=3$ fails in the same way.

The $1_{C}^{2}$ cabling map with $N=2$ does not factor through $b_{n}$ in general either. Further investigations are hindered by the magnitude of the computations required, but these negative results serve well to illustrate the extraordinary nature of the $\rho$-representations in proposition (6.4).

\subsection{On $N=2$ auxiliary spaces, $b_{n}$, and 'The coincidence'}

In (51) we require that $M_{N} \circ f_{b}^{m}(x)$ is also a representation (R say) of $\eta(x)$ for all $x$. Firstly,

$$
\eta: g_{i} \mapsto q+U_{i}
$$


so equation(40) verifies equation(30). We also require $\mathbf{R}\left(U_{1} e_{-} U_{1}-k_{-} U_{1}\right)=0$ for some $k_{-}$(the relation (3) is not sufficient to ensure this). Whenever we find a map, the other question is: Is it faithful?

(8.1) Since

$$
\eta: g_{0} \mapsto \alpha 1+\beta e_{-}
$$

the spectrum of $M_{2}\left(f_{b}^{m}\left(g_{0}\right)\right)$ must be quadratic if the map is to factor through the blob as it stands. By (6.3) this spectrum may be determined from the action on the zero charge sector $\mathcal{P}_{0}$ of $V_{2}^{n}$. The following lists are the eigenvalues with multiplicities in this sector, arranged by standard $T_{n}$-module factor with the 'spine' module on the left and so on.

$M_{2}\left(L_{1}\right):\{1\}$

$M_{2}\left(L_{2}=C_{2}\right):\left\{q^{2}, q^{-2}\right\}$

$M_{2}\left(L_{3}\right):\left\{q^{2}, q^{-2}, q^{-4}\right\} \quad M_{2}\left(C_{3}\right):\left\{1,1, q^{-6}\right\}$

$M_{2}\left(L_{4}\right):\left\{1,1, q^{2}, q^{-4}, q^{-4}, q^{-6}\right\} \quad M_{2}\left(C_{4}\right):\left\{1,1, q^{-4}, q^{-4}, q^{-4}, q^{-12}\right\}$

$M_{2}\left(C_{5}\right):\left\{q^{-4}, q^{-4}, q^{-4}, q^{-4}, q^{-4}, q^{-10}, q^{-10}, q^{-10}, q^{-10}, q^{-20}\right\}$

$M_{2}\left(C_{6}\right):\left\{q^{-8}, . ., q^{-8}, q^{-12}, . ., q^{-12}, q^{-20}, . ., q^{-20}, q^{-32}\right\}$

The pattern for $C_{i}$ will be obvious.

Note, therefore, that $C_{1}, C_{2}, C_{3}$ are the only possibilities here (unless we further specialize to $q$ a root of unity). 'Null' twist $C_{1}$ corresponds to the $m= \pm 1$ case already discussed. For the other cases it remains to check that the candidates for images of the generators obey $U_{1} e_{-} U_{1}=k_{-} U_{1}$ for some scalar $k_{-}$.

(8.2) Example: $M_{2} \circ f_{1}^{2}$. (Let $u_{1}, u_{0}$ denote the canonical preimages along $\eta$ of $U_{1}$ and $e_{-}$.) An elementary calculation finds a value of $k_{-}$for which

$$
M_{2}\left(f_{1}^{2}\left(u_{1} u_{0} u_{1}-k_{-} u_{1}\right)\right)=0
$$

This value then determines that the blob parameter $m=-2$ here.

Since $\operatorname{span}\left(1, g_{1}\right)=\operatorname{span}\left(1, g_{1} g_{1}\right)$ here (and $V_{2}^{n}$ is a faithful $T_{n}$-module) the image of $B_{n}$-braid here is the whole of $T_{n+1}$. Thus (6.3) determines the structure of $V_{2}^{n+1}$ as a $B_{n}$-braid-module. For $n=2$ it is

$$
V_{2}^{3}=1+(2+1)+(2+1)+1
$$

(representing summands by their dimensions) which is the structure as a $T_{3}-$ module. Since we hit the whole of $T_{3}$ equation(52) is the irreducible decomposition with $q$ generic. Let us call the two inequivalent modules here $M^{1}, M^{2}$. The generic simples of $\mathrm{H}(2,2)$, as indexed by their 2-partitions (see $\$ 1$ ), are

\begin{tabular}{|l|c|c|c|c|c|}
\hline 2-partition & $((2))$, & $((1),(1))$ & $(,(2))$ & $\left(\left(1^{2}\right),\right)$ & $\left(,\left(1^{2}\right)\right)$ \\
\hline dimension & 1 & 2 & 1 & 1 & 1 \\
\hline
\end{tabular}

all but the last two of which survive the quotient to $b_{2}$. Note, therefore, that if the $T_{3}$ standard $M^{2}$ breaks up no further (i.e. $q$-generically) it IS a blob representation for $m=-2$, but that $M_{2} \circ f_{1}^{2}$ cannot be a faithful $b_{n}$-module.

For $B_{3}$-braid we have $V_{2}^{4}=1+(3+1)+(2+3+1)+(3+1)+1$ (as a $T_{4}-$ module) cf. $\mathrm{Hecke}_{B}$ :

\begin{tabular}{|l|c|c|c|c|c|c|c|c|c|}
\hline 2-partition & $((3))$, & $((2,1))$, & $\left(\left(1^{3}\right),\right)$ & $((2),(1))$ & $\left(\left(1^{2}\right),(1)\right)$ & $((1),(2))$ & $\left((1),\left(1^{2}\right)\right)$ & $(,(3))$ &.. \\
\hline dimension & $1 *$ & 2 & 1 & $3 *$ & 3 & $3 *$ & 3 & $1 *$ &.. \\
\hline
\end{tabular}


(blob representations indicated with $\mathrm{a}^{*}$ ). Recall that at $m=-2$ blob standards break up as shown in figure 8. In particular at $n=3$

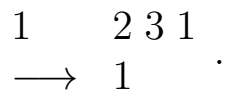

Note that here, and for all $n$, the heads of the $b_{n}$ standards to the right of the $m=-2$ line may indeed be identified with the (generic) irreducible $T_{n+1}$-modules. This is neat, but it follows that none of these representations are faithful.

To summarize the last 2 sections, we have not been successful in generalising the $\rho$-representations. The search for full tilting modules for general $d$ continues, and we report these negative results partly to avoid unnecessary duplication later. More positively, the representations we have found are interesting from the point of view of Yang-Baxter equations in Physics 패, but we will discuss these applications elsewhere.

Acknowledgements Thanks are due to S Dasmahapatra and the members of the Donkin seminar for useful conversations during 1997/98, and EPSRC for funding part of this work under GRJ29923, GRJ55069 and GRM22536. DW has now left the subject, and PPM would like to thank Anton Cox and Steen Ryom-Hansen for encouraging him to finish the paper after an extended hiatus, and also for several useful conversations.

I would like to commend the following recent papers on related topics to the reader: [59, 30].

\section{Appendix}

\section{A On the Bernstein centre $Z(H(n))$ and $Z(\mathbf{H}(n, d))$}

Some of the manipulations of ideals in $\S$ t and thereafter are not trivial. The following mechanical exposition of the Bernstein centre and its image in $Z(\mathrm{H}(n, d))$ may help the reader to see where they come from.

(A.1) Following on from equation([17) define $\hat{g}_{i}=\left[X_{i}, g_{i}\right]$ (thus $\hat{g}_{i}=\left(X_{i}-X_{i+1}\right) g_{i}+$ $\left.\left(q-q^{-1}\right) X_{i+1}\right)$. Then

$$
\begin{gathered}
\hat{g}_{i} X_{j}=X_{\sigma_{i}(j)} \hat{g}_{i} \\
\hat{g}_{i} \hat{g}_{i+1} \hat{g}_{i}=X_{i} g_{i}\left(X_{i+1} g_{i+1}-g_{i+1} X_{i+1}\right) X_{i} g_{i}+\cdots=X_{i} g_{i} X_{i}\left(X_{i+1} g_{i+1}\right) g_{i}-X_{i} g_{i}\left(g_{i+1} g_{i} X_{i} g_{i}\right) X_{i} g_{i}+\cdots \\
=\cdots=X_{i+1} g_{i+1} X_{i} g_{i}^{-1} X_{i+1} g_{i+1}-g_{i+1} X_{i+1} X_{i} g_{i}^{-1} g_{i+1} X_{i+1}+\cdots \\
=X_{i+1} g_{i+1} X_{i}\left(g_{i}-\left(q-q^{-1}\right)\right) X_{i+1} g_{i+1}-g_{i+1} X_{i+1} X_{i}\left(g_{i}-\left(q-q^{-1}\right)\right) g_{i+1} X_{i+1}+\cdots \\
=X_{i+1} g_{i+1} X_{i} g_{i} X_{i+1} g_{i+1}-g_{i+1} X_{i+1} X_{i} g_{i} g_{i+1} X_{i+1}+\cdots=\hat{g}_{i+1} \hat{g}_{i} \hat{g}_{i+1}
\end{gathered}
$$

Let $K$ be our ground ring (an integral domain), $K\left[X_{-}\right]$the ring of polynomials in the $X_{i} \mathrm{~s}$, and $K\left(X_{-}\right)$the field of fractions. Note that we can write

$$
\alpha_{i} \hat{g}_{i}=g_{i}+\beta_{i}
$$


where both $\alpha_{i}$ and $\beta_{i}$ lie in $K\left(X_{-}\right)$. (NB, in our quotient $\Psi_{d}$, the image of $K\left[X_{-}\right]$ itself generically, but not always, contains $\alpha_{i}$ and $\beta_{i}$. For example, when $d=1$ and $q^{2}=1$ then $X_{i}-X_{i+1}$ is not invertible.) It then follows from equation(53) that any element of the extension of $H(n)$ by $K\left(X_{-}\right)$can be expressed in the form

$$
h=\sum_{w \in \mathfrak{B}_{n}} c_{w} \hat{g}_{w}
$$

where $c_{w} \in K\left(X_{-}\right)$and $\hat{g}_{w}$ is obtained by putting hats on the generators in $w$. Indeed the extension may be decomposed as

$$
\bigoplus_{w \in \mathfrak{B}_{n}} K\left(X_{-}\right) \hat{g}_{w}
$$

(an induction on the usual length function on $\mathfrak{B}_{n}$ ). Suppose $h$ has at least one $w \neq 1$ with $c_{w} \neq 0$ (i.e. $h \notin K\left(X_{-}\right)$subalgebra). Then there is at least one $i$ such that $w(i) \neq i$ (under the obvious generalisation of the $\sigma_{i}$ action in equation(53)) and

$$
h X_{i}=\sum_{w} c_{w} \hat{g}_{w} X_{i}=\sum_{w} c_{w} X_{w(i)} \hat{g}_{w}
$$

so the $\hat{g}_{w}$ component of $h X_{i}-X_{i} h$ is $c_{w}\left(X_{w(i)}-X_{i}\right) \neq 0$. Thus $Z_{K\left[X_{-}\right]}(H(n))=$ $K\left[X_{-}\right] \supseteq Z(H(n))$. But with $c \in K\left[X_{-}\right]$, then $\hat{g}_{w} c-c \hat{g}_{w}=\left(c^{w}-c\right) \hat{g}_{w}$ so $Z(H(n))=$ $X^{\Sigma}$ as Bernstein says.

(A.2) Naturally $Z(\mathrm{H}(n, d)) \supseteq \Psi_{d}\left(X^{\Sigma}\right)$, depending in principle on the ground ring. The argument above mostly works in this case (to show equality), although the possible specialisations of the ground ring become more restricted $\left(\lambda_{i} \neq 0\right.$, plus the restrictions already mentioned, for example). Note also that $\Psi_{d}\left(K\left[X_{-}\right]\right)$is finite dimensional. This makes it interesting to study $\Psi_{d}\left(X^{\Sigma}\right)$ - an algebra which is, in a sense, more complicated that $X^{\Sigma}$ itself. For example, $\mathrm{H}(2,1) \cong H_{n}$ and $\operatorname{dim}\left(Z\left(H_{2}\right)=\right.$ $\left.H_{2}\right)=2$, so $\operatorname{dim}\left(\Psi_{1}\left(X^{\Sigma}\right)\right)=2$. Here a basis for $\Psi_{1}\left(X^{\Sigma}\right)$ is $\left\{1, X+X_{2}=\lambda_{1}\left(1+g_{1}^{2}\right)\right\}$ (note that this example illustrates the problem with $q^{2}=1$ ).

For another example, recall that $\operatorname{dim}(Z(\mathrm{H}(2,2)))=5$ generically, so $\operatorname{dim}\left(\Psi_{1}\left(X^{\Sigma}\right)\right)=$ 5. Here a basis for $\Psi_{2}\left(X^{\Sigma}\right)$ is $\left\{1, X+X_{2}, X X_{2}, X^{2}+X_{2}^{2},\left(X+X_{2}\right) X X_{2}\right\}$ (this is not supposed to be obvious!). Another basis, convenient for comparison with the basis of $\mathrm{H}(2,2)$, is

$$
\left\{1, X+X_{2}, X X_{2},\left(X+X_{2}-\left(\lambda_{1}+\lambda_{2}\right)\right) g_{1},\left(X X_{2}-\lambda_{1} \lambda_{2}\right) g_{1}\right\}
$$

(A.3) The set of monomial symmetric polynomials in two variables $X_{1}, X_{2}$ (a basis for $Z(H(2)))$ may be indexed by the set $\Lambda^{2}$ of Young diagrams of not more than two rows (write $m^{a}=\left(X^{a}\right)^{\Sigma}$, then $m^{(0)}=1$ and $m^{(1)}=X_{1}+X_{2}$, and so on). NB, The set of such polynomials in which the degree of no individual variable exceeds $d-1$ is not a basis for $\Psi_{d}\left(X^{\Sigma}\right)$ in general, as we see from the examples above.

\section{References}

[1] S Ariki, On the decomposition numbers of the Hecke algebra of $G(m, 1, n)$, Journal of Mathematics of Kyoto University 36 (1996), 789-808. 
[2] S. Ariki and K. Koike, A Hecke algebra of $(\mathbb{Z} / r \mathbb{Z})$ ? $S_{n}$ and construction of its irreducible representations, Adv. in Math. (1994), 216-243.

[3] S Ariki, T Terasoma, and H Yamada, Schur-Weyl reciprocity for the Hecke algebra of $(z / r z)$ ? $s_{n}$, J Algebra 178 (1995), 374-390.

[4] R J Baxter, Exactly solved models in statistical mechanics, Academic Press, New York, 1982.

[5] R J Baxter, S B Kelland, and F Y Wu, Equivalence of the Potts model or Whitney polynomial with an ice-type model, J Phys A 9 (1976), 397-406.

[6] R Behrend and P A Pearce, Boundary weights for Temperley-Lieb and dilute Temperley-Lieb models, Int J Mod Phys B 11 (1997), 2833-2847.

[7] D J Benson, Representations and cohomology I, Cambridge, 1995.

[8] J S Birman, Braids, links and mapping class groups, vol. 82, Annals of Mathematics Studies, Princeton University Press, Princeton NJ, 1975.

[9] N Bourbaki, Groupes at algebres de Lie, vol. 4-6, Masson, 1981.

[10] M. Broué and G. Malle, Zyklotomische heckealgebren, Astérisque 212 (1993), 119-189.

[11] I Cherednik, A unification of Knizhnik-Zamolodchikov and Dunkl operators via affine Hecke algebras, Invent Math 106 (1991), 411-431.

[12] E. Cline, B. Parshall, and L. Scott, Finite dimensional algebras and highest weight categories, J. Reine Angew. Math. 391 (1988), 85-99.

[13] E Cline, B Parshall, and L Scott, Generic and q-rational representation theory, Publ RIMS 35 (1999), 31-90.

[14] P M Cohn, Algebra, vol. 2, Wiley, New York, 1982.

[15] A G Cox, J J Graham, and P P Martin, The blob algebra in positive characteristic, City University preprint 2001, submitted to J Algebra.

[16] A G Cox, P P Martin, and S Ryom-Hansen, Virtual algebraic Lie theory, in preparation.

[17] E Date, M Jimbo, T Miwa, and M Okado, Adv Stud Pure Math 16 (1988), 17.

[18] T Deguchi, J Phys Soc Japan 58 (1989), 3441.

[19] R Dipper, G James, and A Mathas, The $(Q, q)$-Schur algebra, preprint (1999).

[20] V Dlab and C M Ringel, A construction for quasi-hereditary algebras, Compositio Mathematica 70 (1989), 155-175.

[21] S. Donkin, On tilting modules for algebraic groups, Math. Z. 212 (1993), 39-60.

[22] — The q-Schur algebra, LMS Lecture Notes Series, vol. 253, Cambridge University Press, 1998. 
[23] W Fulton and J Harris, Representation theory, Springer, 1991.

[24] A M Garsia and T J McLarnan, Relations between Youngs natural and the Kazhdan-Lusztig representations of $s_{n}$, Advances in Mathematics 69 (1988), 32-92.

[25] R Goodman and N R Wallach, Representations and invariants of the classical groups, Cambridge, 1998.

[26] J. J. Graham and G. I. Lehrer, Diagram algebras, Hecke algebras and decomposition numbers at roots of unity, preprint.

[27] - The representation theory of affine Temperley-Lieb algebras, L'Enseignement Mathématique 44 (1998), 173-218.

[28] J A Green, Polynomial representations of $G L_{n}$, Springer-Verlag, Berlin, 1980.

[29] R M Green, Tabular algebras and their asymptotic versions, J Algebra (to appear) (2001).

[30] I Grojnowski, Affine $\hat{s l}_{p}$ controls the modular representation theory of the symmetric group and related Hecke algebras, math.RT/9907129, 1999.

[31] M Hamermesh, Group theory, Pergamon, Oxford, 1962.

[32] P N Hoefsmit, Representations of Hecke algebras of finite groups with BN pairs of classical type, Ph.D. thesis, University of British Columbia, 1974.

[33] J E Humphreys, Reflection groups and Coxeter groups, Cambridge University Press, 1990.

[34] G D James and A Kerber, The representation theory of the symmetric group, Addison-Wesley, London, 1981.

[35] J.C. Jantzen, Representations of algebraic groups, Academic Press, 1987.

[36] M Jimbo, A q-difference analogue of $u(g)$ and the Yang-Baxter equation, Lett Math Phys 10 (1985), 63-69.

[37] V F R Jones, A quotient of the affine Hecke algebra in the Brauer algebra, L'Enseignement Mathématique 40 (1994), 313-344.

[38] A Joseph, Quantum groups and their primitive ideals, Springer-Verlag, 1995.

[39] M Kashiwara, T Miwa, and E Stern, Decomposition of q-deformed Fock spaces, q-alg/9508006, 1995.

[40] D Kazhdan and G Lusztig, Representations of coxeter groups and Hecke algebras, Inventiones Math. 53 (1979), 165-184.

[41] S.S.F. Lambropoulou, Solid torus links and Hecke algebras of B type, Proceedings of the Quantum Topology, World Scientific, Singapore, 1994, p.225.

[42] A Lascoux, B Leclerc, and J-Y Thibon, Hecke algebras at roots of unity and crystal bases of quantum affine algebras, Commun Math Phys 181 (1996), 205263. 
[43] G Lusztig, Affine Hecke algebras and their graded version, J Am Math Soc 2:3 (1989), 599-685.

[44] I Macdonald, Symmetric functions and Hall polynomials, Oxford, 1979.

[45] W Magnus, A Karras, and S Solitar, Combinatorial group theory, Wiley, 1966.

[46] P P Martin, Analytic properties of the partition function for statistical mechanical models, J Phys A 19 (1986), 3267-3277.

[47] _ Block spin transformations in the operator formulation of twodimensional Potts models, J Phys A 22 (1989), 3991-4005.

[48] _ String-like lattice models and Hecke algebras, J Phys A 22 (1989), 3103-3112.

[49] _ Potts models and related problems in statistical mechanics, World Scientific, Singapore, 1991.

[50] P P Martin and D Levy, Hecke algebra solutions to the reflection equations, Journal of Physics A 27 (1994), L521-L526.

[51] P P Martin and H Saleur, On an algebraic approach to higher dimensional statistical mechanics, Commun. Math. Phys. (1993), no. 158, 155-190.

[52] _ The blob algebra and the periodic Temperley-Lieb algebra, Lett. Math. Phys. (1994), no. 30, 189-206.

[53] P P Martin and D Woodcock, On quantum spin-chain spectra and the structure of Hecke algebras and q-groups at roots of unity, J Phys A 31 (1998), 1013110154.

[54] _ On the structure of the blob algebra, J Algebra 225 (2000), 957-988.

[55] P P Martin, D Woodcock, and D Levy, A diagrammatic approach to Hecke algebras of the reflection equation, J Phys A 33 (2000), 1265-1296.

[56] A Mathas, Canonical bases and the decomposition matrices of Ariki-Koike algebras, preprint (1996).

[57] V Pasquier and H Saleur, Nucl Phys B 330 (1990), 523.

[58] J D Rogawski, On modules over the Hecke algebra of a p-adic group, Invent Math 79 (1985), 443-465.

[59] S Sakamoto and T Shoji, Schur-Weyl reciprocity for Ariki-Koike algebras, J Algebra 221 (1999), 293-314.

[60] W. Soergel, Charakterformeln für Kipp-Moduln über Kac-Moody-Algebren, Representation Theory 1 (1997), 115-132.

[61] _ Kazhdan-Lusztig polynomials and a combinatoric for tilting modules, Representation Theory 1 (1997), 83-114.

[62] R P Stanley, Enumerative combinatorics, Cambridge, 1997. 
[63] D Stanton and D White, Constructive combinatorics, UTM, Schwinger, New York, 1986.

[64] H N V Temperley and E H Lieb, Proceedings of the Royal Society A 322 (1971), $251-280$.

[65] T tom Dieck, Symmetrische brucken und knotentheorie zu den Dynkindiagrammen vom type B, J Reine angew Math 451 (1994), 71-88.

[66] A M Vershik and A Y Okunkov, An inductive method of expounding the representation theory of symmetric groups, Russian Mathematical Surveys 51 (1996), 1237-1239. 